\title{
Payments on Digital Platforms: Resiliency, Interoperability and Welfare
}

by Jonathan Chiu ${ }^{1}$ and Tsz-Nga Wong ${ }^{2}$

${ }^{1}$ Banking and Payments Department

Bank of Canada, Ottawa, Ontario, Canada K1A 0G9

${ }^{2}$ Federal Reserve Bank of Richmond

jchiu@bankofcanada.ca, Russell.Wong@rich.frb.org

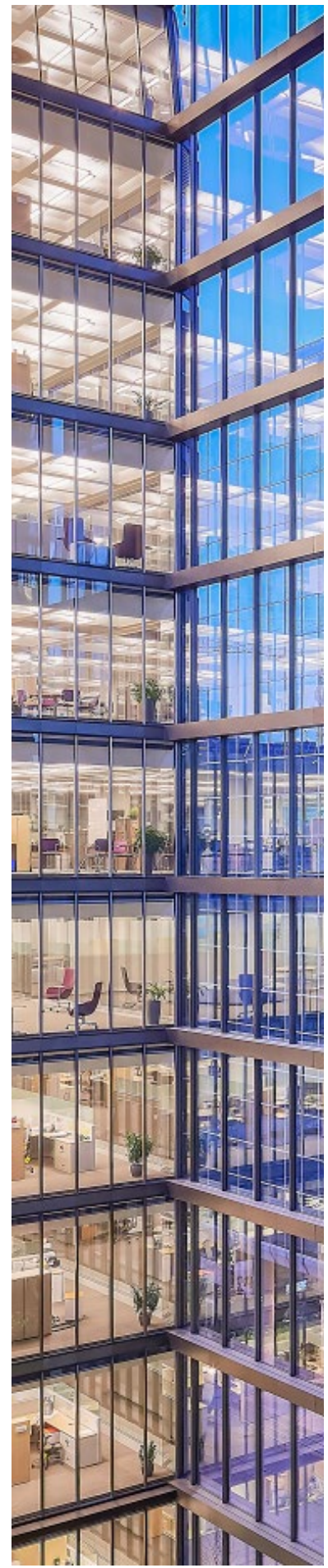

Bank of Canada staff working papers provide a forum for staff to publish work-in-progress research independently from the Bank's Governing Council. This research may support or challenge prevailing policy orthodoxy. Therefore, the views expressed in this paper are solely those of the authors and may differ from official Bank of Canada views. No responsibility for them should be attributed to the Bank. 


\section{Acknowledgements}

We have benefited from the comments and suggestions of participants, especially Charlie Kahn,

at conferences and seminars. The views expressed here do not necessarily reflect those of the

Bank of Canada, the Federal Reserve Bank of Richmond, or the Federal Reserve System. 


\section{Abstract}

Digital platforms, such as Alibaba and Amazon, operate an online marketplace to facilitate transactions. This paper studies a platform's business model choice between accepting cash and issuing tokens, as well as the implications for welfare, resiliency, and interoperability. A cash platform free rides on the existing payment infrastructure and profits from collecting transaction fees. A token platform earns seigniorage, albeit bearing the costs of setting up the system and holding reserves to mitigate the cyber risk. Tokens earn consumers a return, insulating transactions from the liquidity costs of using cash, but also expose them to the remaining cyber risk. The platform issues tokens if the interest rate is high, the platform scope is large, and the cyber risk is small. Unbacked floating tokens with zero transaction fees or interest-bearing stablecoins can implement the equilibrium business model, which is not necessarily socially optimal because the platform does not internalize its impacts on offplatform activities. The model explains why Amazon does not issue tokens but Alipay issues tokens circulatable outside its Alibaba platforms. Regulations such as a minimum reserve requirement can reduce welfare.

Topics: Digital currencies and fintech; Monetary policy; Payment clearing and settlement systems

JEL codes: E, E4,E5, L, L5 


\section{Introduction}

Recent years have witnessed the emergence of private digital tokens issued by social and economic platforms to facilitate transactions among users in a variety of contexts. Early digital tokens were issued by social media networks to allow platform users to conduct peer-to-peer transfers. ${ }^{1}$ In the past few years, many blockchain-based platforms have launched utility coins circulating in their distributed networks as the only acceptable means of payment. ${ }^{2}$ In China, Alibaba's and WeChat's digital wallets, which allow holders to accumulate redeemable, interest-bearing balances for online and point-of-sale transactions, have dominated the retail payments system. In the United States, retail giants Walmart and Amazon have also recently applied for patents related to cryptocurrencies.

Private tokens, especially those issued by major platforms with a large user base, could reshape the retail payment landscape and influence the functioning of the monetary system. The influence of private tokens has been an important theme of policy discussion. ${ }^{3}$ According to a central banks survey by Auer, Cornelli and Frost (2020), the announcement of Facebook's Libra in 2019 was a tipping point to urge the development of central bank digital currencies (CBDCs). The share of central banks that are likely to issue a retail CBDC in one to six years doubled in 2019 to $20 \%$.

Within this context, our paper studies the business model choice between running a cash platform and a token platform, as well as its welfare and policy implications. What trade-offs does a platform face when choosing between accepting cash and issuing its own tokens? How does a token platform design features such as transaction fees, token interests, and reserves held for redemption? How are the token prices determined? Is the platform's choice socially optimal? What are the implications for payment resiliency and interoperability? What is the scope for regulation?

The key trade-off between the two business models is as follows. A cash platform makes profits by charging fees on platform transactions. However, the platform's ability to extract consumer surplus is constrained by consumers' cash holdings. In particular, the platform cannot charge profit-maximizing fees when consumers' cash holdings are tight due to high inflation (or any other costs associated with using cash). By issuing tokens, a platform can insulate platform transactions from the costs associated with cash. ${ }^{4}$ Issuing tokens also provides the platform with an option to use token sales, i.e., the seigniorage, as an alternative source of revenue. Unlike cash, tokens can pay interest to the holders to boost their demand. However, tokens can lose transaction value when the platform ceases to operate (e.g., due to cyber attacks), and hence tokens, unlike cash, are subject to default risk. To support the transaction value of tokens, the platform may need to hold reserves in liquid low-yield assets, such as cash, for consumers' redemption.

\footnotetext{
${ }^{1}$ Early examples are Tencent QQ, Second Life Linden, and Facebook Credits. In June 2019, Facebook announced its intention to introduce Libra, a blockchain-based stablecoin.

${ }^{2}$ Examples are Ethereum tokens, used in the Ethereum system, as well as Filecoin and Sia, used in cloud storage networks.

${ }^{3}$ See Kahn, Rivadeneyra and Wong (2020b) for a summary of the policy discussion about the private and public provision of digital currencies.

${ }^{4}$ This cash constraint was a driving force behind the development of some digital platforms. For example, Alibaba launched its own payments product, Alipay, in 2004 partly due to the inefficiency of the "cash-on-delivery" payment method on its Taobao platform.
} 
We find that the optimal platform design features zero reserves. This is because a token platform can support the transaction value of tokens by simply engineering a sufficiently high yield of tokens (e.g., fast-appreciating token prices). The platform also profits from selling tokens at fast-appreciating prices. The trade-off is that the platform has to limit the quantity of tokens sold or keep buying back tokens in order to sustain the appreciation. There are multiple ways to implement the optimal yield of tokens. For example, the platform can charge a zero transaction fee or pay interest on tokens.

Given the above trade-off, when would a platform choose to issue tokens instead of accepting cash? When inflation is not too high, using cash is optimal, especially when setting up a token system is costly. A platform chooses to issue tokens when inflation is sufficiently high (so that the cash holding is tight), when the platform is sufficiently resilient to cyber attacks (so that tokens are expected to circulate longer) and when its market share is sufficiently large (so that transaction fees are not a good way to collect revenue). Interestingly, the platform's choice of business model is not necessarily socially optimal because it ignores the impact on consumers' welfare. Depending on parameter values, both cash and token platforms can be suboptimally adopted. For example, the over-adoption of token platforms happens when the platform does not internalize the negative impacts of rejecting cash on non-platform consumption.

Could policy intervention improve social welfare? When the platform's business model choice is suboptimal, a simple tax/subsidy scheme, if feasible, can induce the platform to make the optimal decision. However, conditional on the adoption of a token system, its design can still be suboptimal relative to the first-best allocation. Could imposing reserve requirements be welfare improving? ${ }^{5}$ We find that it is optimal for a token platform to minimize its reserve holdings, and hence a reserve regulation is typically welfare reducing.

Our paper contributes to the young but fast-growing literature exploring the economics of digital currencies. A large amount of research focuses on understanding the design and functioning of cryptocurrencies, such as bitcoin. This literature can be broadly divided into three lines of research. The first line aims at understanding the pricing of cryptocurrencies, e.g., Biais, Bisiere, Bouvard, Casamatta and Menkveld (2020), Choi and Rocheteau (2020), and Schilling and Uhlig (2019). The second line examines the functioning and robustness of the blockchain, e.g., Biais, Bisiere, Bouvard and Casamatta (2019), Eyal and Sirer (2014), Saleh (2020), Chiu and Koeppl (2019), and Pagnotta (2020). The third line focuses on mining activities and the determination of fees, e.g., Huberman, Leshno and Moallemi (2017) and Easley, O'Hara and Basu (2019).

There are important differences between general-purpose cryptocurrencies and platform-based tokens. Unlike general-purpose cryptocurrencies, platform-based tokens are mainly used for activities on the platform, and the platform controls whether or not to circulate the tokens outside the platform, subject to regulatory and technical restrictions. Also, platform-based tokens are often

\footnotetext{
${ }^{5}$ For example, in China, the $\mathrm{PBoC}$ recently imposed $100 \%$ reserve requirements on third-party payment groups. According to a report by Financial Times, Alipay and Tencent were poised to lose around $\$ 1$ billion in combined annual revenue (source: https://tinyurl.com/yax3hjjf).
} 
redeemable in national currency or platform goods. ${ }^{6}$ Therefore, the value of platform-based tokens depends on the functioning of the issuing platform as well as the issuer's commitment to future actions, such as redemption, investment, and reserve holdings. Finally, the token prices and fees are determined by a forward-looking profit-maximizing platform like a durable-goods monopoly. The monopoly power of the platform comes from its network effect.

A more closely related research area focuses on the design of utility tokens issued by platforms and the value of initial coin offerings as a mode of financing, e.g., Catalini and Gans (2018), Cong, Li and Wang (2020), Howell, Niessner and Yermack (2020), Garratt and Van Oordt (2019), Sockin and Xiong (2020), and You and Rogoff (2019). Gans and Halaburda (2015 Apr 20) study the design of private digital currencies from a platform-management perspective and explain why a profit-maximizing platform may choose to limit the functionality of digital currencies. This stream of research typically focuses on partial-equilibrium analysis and thus does not study macro implications of platform-based tokens. Our paper complements this literature by highlighting the interaction between on-platform and off-platform activities in a general equilibrium setting.

Some recent studies develop general equilibrium models to investigate the macro implications of issuing central bank digital currencies, e.g., Andolfatto (2018), Barrdear and Kumhof (2016), Chiu and Wong (2014), Chiu, Davoodalhosseini, Jiang and Zhu (2019), Davoodalhosseini (2018), Fernández-Villaverde, Sanches, Schilling and Uhlig (2020), Keister and Sanches (2019) and Williamson (2019). Zhu and Hendry (2019) study the competition between national currency and general-purpose electronic money. To the best of our knowledge, this paper is the first one to study the welfare and policy implications of issuing platform-based digital tokens in a general equilibrium setting.

Cyber risk and security issues have been investigated in the payment literature, for example, Kahn and Roberds (2008), and Kahn, Rivadeneyra and Wong (2020a) study the trade-off between security and convenience. We explore how platform security influences payment design in a general equilibrium environment.

Our model is based on Lagos and Wright (2005), which provides a tractable framework for us to study the joint determination of the transaction values of cash and tokens and the general equilibrium effects of a platform's payment choice. See also the survey in Kahn and Roberds (2009). Recent models of payment systems building on the Lagos-Wright framework include Li (2011) and Monnet and Roberds (2008). Chiu and Wong (2015) adopt a mechanism design approach to compare the essential payment features of digital currency with cash.

The rest of the paper is organized as follows. Section 2 describes the basic environment. Sections 3 and 4 examine, respectively, a cash platform and a token platform. Section 5 studies the equilibrium business model and its welfare implications. Section 6 discusses the effects of token platform regulation. Section 7 considers an extension with endogenous interoperability and pro-

\footnotetext{
${ }^{6}$ In other words, government-issued fiat currencies and cryptocurrencies, like bitcoin, are outside money, while redeemable platform-based tokens are inside money. But unlike typical inside money, such as deposit, platform-based tokens do not involve any maturity transformation.
} 
vides examples calibrated to Amazon and Alibaba. Section 8 concludes. All proofs are provided in the Appendix.

\section{Environment}

Our model is based on the alternating competitive markets of Lagos and Wright (2005). Time is indexed by $t \in \mathbb{N}_{0}$. Each period is divided into two stages. In stage 1, competitive markets for retail goods open, and consumption opportunities arise. In stage 2, agents trade assets and numeraires. The economy starts in stage 2 of period 0 . There are three types of agents: merchants who can produce retail goods in stage 1 and consume numeraires in stage 2, consumers who consume and produce numeraires in stage 2 and consume retail goods if opportunities arise in stage 1 , and a platform that produces nothing but facilitates meetings between consumers and merchants in stage 1 as explained below. The population of consumers is normalized to one. When consumption opportunities arise, there is a chance that the consumer can find a suitable merchant on or off the platform. Consumers and the platform discount across periods with a factor $\beta=1 /(1+r)$, where $r>0$. Merchants live for a period with new merchants entering at the beginning of stage 1 . Starting from period 1, a cyber shock hits the platform with probability $\gamma$ in stage 2 , after which the platform will cease to exist in the next period. A low $\gamma$ captures that the platform is resilient to constant cyber attacks in the background.

The preferences of merchants in period $t$ are given by

$$
-q_{t}+c_{t}
$$

where $q_{t}$ is the retail goods produced in stage 1 , and $c_{t}$ is the numeraire consumed in stage 2 . The preferences of consumers are given by

$$
\mathbb{E} \sum_{t=0}^{\infty} \beta^{t}\left\{I_{q t} U\left(q_{t}\right)+I_{y t} U\left(y_{t}\right)+u\left(c_{t}, h_{t}\right)\right\},
$$

where $U$ is the consumer's utility in stage 1 such that $U(k)=k^{1-\eta} /(1-\eta)$ for $k=q$, $y$, where $\eta \in(0,1)$, and $u$ is the consumer's utility in stage 2 such that $u(c, h)=\bar{u}\left[c_{t}^{\lambda}+\varphi\left(\bar{h}-h_{t}\right)^{\lambda}\right]^{1 / \lambda}$, where $h_{t} \in[0, \bar{h}]$ is the consumer's production of numeraires in stage $2, \lambda \in[-\infty, 1], \varphi>0$, $\bar{u}=\left[1+\varphi^{1 /(1-\lambda)}\right]^{-(1-\lambda) / \lambda}$ and $\bar{h}$ is sufficiently large. $I_{q t}$ and $I_{y t}$ are indicator variables capturing the iid consumption opportunity. Conditional on no cyber shocks taking place, with probability $\sigma(1-\alpha)$, a consumption opportunity arises off the platform (i.e., $I_{q}=1, I_{y}=0$ ); with probability $\sigma \alpha$, it arises on the platform (i.e., $I_{q}=0, I_{y}=1$ ); with probability $1-\sigma$ it does not occur (i.e., $\left.I_{q}=0, I_{y}=0\right)$. After a cyber shock, with probability $\sigma(1-\alpha)$, it arises off the platform (i.e., $I_{q}=1, I_{y}=0$ ); with probability $1-\sigma(1-\alpha)$ it does not occur (i.e., $\left.I_{q}=0, I_{y}=0\right) .{ }^{7}$ Thus, a higher $\alpha$ means the consumption opportunities are more likely to be met via the platform. The

\footnotetext{
${ }^{7}$ The stochastic structure of the model is important for generating the endogenous linkage between platform and non-platform activities in stage 1. This assumption also simplifies the analysis by making agents ex-ante homogeneous.
} 
preferences of the platform are given by

$$
x_{0}+\sum_{t=1}^{\infty}(1-\gamma)^{t-1} \beta^{t}\left[(1-\gamma) x_{1 t}+\gamma x_{0 t}\right],
$$

where $x_{0 t}\left(x_{1 t}\right)$ is the platform's consumption of numeraires in stage 2 of $t$ if a cyber has (not) hit, and $x_{0}$ is the initial platform's consumption.

Agents can only observe the actions and outcomes of their trades. Consumers are anonymous and cannot commit. There is no enforcement or record-keeping technology. As a result, consumers cannot borrow to finance their consumption because they would renege on their promise to repay their debt. This generates the need for a medium of exchange. In this economy, there are perfectly divisible storable objects called cash that can be used as a medium of exchange. The supply of cash is maintained exogenously by a central bank's lump-sum transfers to consumers in stage 2 , such that the real price of cash in period $t$ is $\phi_{t}$. We assume an exogenous monetary policy that fixes the inflation rate at $\bar{\mu}=\phi_{t} / \phi_{t+1}$.

We interpret the platform as an online marketplace, such as Amazon and Alibaba, where the platform does not act as a buyer/seller but intermediates goods and payments between consumers and merchants who do not have direct contacts among themselves. This role allows the platform to dictate the means of payments acceptable by the merchants on the platform. ${ }^{8}$ Also, note that the amount paid by the consumer is not necessarily equal to the amount received by the merchant. The difference is the transaction fee charged by the platform. In the next section, we will focus on a cash platform that allows its users to use cash to transact on the platform. To trade on the cash platform, in stage 1, consumers need to acquire cash balances in the previous stage 2 by selling their production of numeraires. On the platform, consumers are subject to a CIA constraint. In addition, the platform can charge a proportional transaction fee that is proportional to the transaction value on the platform and is paid in cash. ${ }^{9}$ Later we will extend the model to study a token platform, which issues an additional medium of exchange called tokens. We will then study the equilibrium business model of the platform and its welfare implications.

We expect that, as long as the consumption shocks are not perfectly predictable, consumers will still hold both tokens and cash and the basic results hold.

${ }^{8}$ Specifically, in stage 1, (1) the platform monitors and controls only merchants' flow of tokens; (2) the physical changing hands of cash is only possible between an agent and platform but not among agents on the platform; (3) consumers on the platform can find each other and trade among themselves privately, if they want to. In this environment, due to (1) and (2), the platform can restrict the type of payment instruments acceptable by the merchants and impose fees on their transactions. However, due to (3), the platform cannot monitor buyers or prohibit side trades among themselves. So lump sum fees will be infeasible as consumers can avoid them by side-trades. These are reasonable assumptions for an e-commerce platform built to connect anonymous buyers with online merchants. Merchants cannot communicate with buyers easily outside the supervision of the platform. But there are many ways for anonymous consumers to communicate among themselves outside the supervision of the platform.

${ }^{9}$ Unlike lump-sum fees, proportional fees are not subject to side-trading; see Chiu and Wong (2015) for details. It is now well known in the industrial organization literature that proportional fees are more profitable than lump-sum fees in general environments with heterogeneity; see Shy and Wang (2011). Thus, we do not pursue the analysis of lump-sum fees in this paper. 


\section{Cash platform}

A cash platform allows consumers to use cash as the medium of exchange and makes profit solely from charging the transaction fee $\tau_{t}$ on the (post-fee) transaction value on the platform. We solve the merchant's and consumer's problems backward by first considering stage 1 of period $t$ and then stage 2 of period $t-1$.

\subsection{Merchant's problem}

In the competitive market of stage 1 , merchants choose $q_{t}$ units of retail goods to be sold on the platform and $y_{t}$ units of retail goods to be sold off the platform, taking as given the nominal price $P_{t}$ of retail goods, which is the same on or off the platform. The zero-profit condition implies $-q_{t}+\phi_{t} P_{t} q_{t}=0=-y_{t}+\phi_{t} P_{t} y_{t}$, where $-q_{t}$ is the cost of producing retail goods, and $\phi_{t} P_{t} q_{t}$ is the real value of cash receipt. Hence, the equilibrium price of retail goods is $P_{t}=1 / \phi_{t}$.

\subsection{Consumer's problem}

At the beginning of stage 1 in period $t$, the value function of holding $M$ units of cash is

$$
\begin{gathered}
V_{t}(M)=\max _{q_{t}, y_{t}}\left\{\begin{array}{c}
\sigma(1-\alpha)\left[U\left(q_{t}\right)+W_{t}\left(M-P_{t} q_{t}\right)\right] \\
+\sigma \alpha\left\{U\left(y_{t}\right)+W_{t}\left(M-\frac{P_{t} y_{t}}{1-\tau_{t}}\right)\right\} \\
+(1-\sigma) W_{t}(M)
\end{array}\right\} \text { s.t. } \\
M \geq P_{t} q_{t} \\
M \geq \frac{P_{t} y_{t}}{1-\tau_{t}}
\end{gathered}
$$

where $q_{t}$ is the quantity bought off the platform, $y_{t}$ is the quantity of retail goods bought on the cash platform, $W_{t}$ is the continuation value function in stage 2 , and (2) and (3) are the associated CIA constraints for non-platform and platform transactions respectively. With probability $1-\sigma$, the consumer does not need anything in stage 1 , and he brings $M$ units of cash to stage 2 . With probability $\sigma(1-\alpha)$, the consumer pays some merchants off the platform $P_{t} q_{t}$ units of cash for $q_{t}$ units of retail goods, bringing the remaining $M-P_{t} q_{t}$ units of cash to stage 2 . With probability $\sigma \alpha$, the consumer pays on the cash platforms $P_{t} y_{t} /\left(1-\tau_{t}\right)$ units of cash for $y_{t}$ units of retail goods, of which $P_{t} y_{t}$ is paid to the merchants and $\tau_{t} P_{t} y_{t} /\left(1-\tau_{t}\right)$ to the platform as the transaction fees. ${ }^{10}$

At the beginning of stage 2 of period $t-1$, the value function of a consumer holding $M$ units of cash is

$$
W_{t-1}(M)=\max _{\left\{c_{t}, h_{t}, m_{t}\right\} \in \mathbb{R}_{+}^{3}}\left\{u\left(c_{t-1}, h_{t-1}\right)+\beta V_{t}\left(M_{t}\right)\right\} \text {, s.t. }
$$

\footnotetext{
${ }^{10}$ Consistent with off-the-platform trades, we use $P_{t} y_{t}$ to denote the amount that on-platform merchants receive. Denote $D_{t}$ as the total payment the consumer pays, then the on-platform merchant receives $\left(1-\tau_{t}\right) D_{t}=P_{t} y_{t}$. Thus, the transaction fee the platform receives is $\tau_{t} D_{t}=\tau_{t} P_{t} y_{t} /\left(1-\tau_{t}\right)$.
} 


$$
c_{t-1}+\phi_{t-1} M_{t}=h_{t-1}+\phi_{t-1} M+\Upsilon_{t}
$$

where $\Upsilon_{t}$ is the real lump-sum transfer from the central bank needed to implement the price path $\phi_{t-1}=\bar{\mu} \phi_{t}$. Applying the envelope theorem to (4) and (1), we obtain

$$
\frac{d W_{t}(M)}{d M}=\phi_{t}, \frac{d V_{t}(M)}{d M}=\phi_{t}\left[\begin{array}{l}
1+\sigma(1-\alpha)\left\{U^{\prime}\left(\frac{M_{t}}{P_{t}}\right)-1\right\}_{+} \\
+\sigma \alpha\left\{\left(1-\tau_{t}\right) U^{\prime}\left[\left(1-\tau_{t}\right) \frac{M_{t}}{P_{t}}\right]-1\right\}_{+}
\end{array}\right],
$$

where $\{X\}_{+} \equiv \max \{X, 0\}$. ${ }^{11}$ The first-order condition of (4) with respect to $M_{t}$ implies that

$$
i=\sigma(1-\alpha)\left\{U^{\prime}\left(m_{t}\right)-1\right\}_{+}+\sigma \alpha\left\{\left(1-\tau_{t}\right) U^{\prime}\left[\left(1-\tau_{t}\right) m_{t}\right]-1\right\}_{+},
$$

where $i \equiv(1+r) \phi_{t-1} / \phi_{t}-1=(1+r) \bar{\mu}-1>0$ is the nominal interest rate and $m_{t} \equiv \phi_{t} M_{t}$ is the real balances. The rate $i$ on the LHS of (6) captures the marginal (opportunity) cost of holding cash, which is the nominal interest forgone. The first and second terms on the RHS of (6) capture the marginal returns of spending cash off the platform and on the platform, respectively. These terms are positive whenever holding cash helps relax the binding CIA constraints, (2) and (3). Finally, note that the aggregate stage- 2 utility is a constant and equal to $w_{t} \equiv \int u\left[c_{t}(A), h_{t}(A)\right] d F_{t}(A)+$ $\sigma \alpha q_{t}+\sigma(1-\alpha) y_{t}+x_{t}=\bar{h}$ for all $t$, where $c_{t}(A)$ and $h_{t}(A)$ are the solution to (11) given $A$ and $F_{t}(A)$ is the distribution of $A$ in the beginning of stage 2 at $t$.

We first derive the consumer's choices $\left(q_{t}, y_{t}, m_{t}\right)$ as a function of $\left(\tau_{t}, i\right)$, which is summarized by Lemma 1.

\section{Lemma 1 Define}

$$
\begin{aligned}
\tau_{C}(i) & \equiv 1-\left[1+\frac{i}{\sigma(1-\alpha)}\right]^{-1 /(1-\eta)}, \\
m\left(\tau_{t}\right) & \equiv\left(1+\frac{i}{\sigma}\right)^{-1 / \eta}\left[1-\alpha+\alpha\left(1-\tau_{t}\right)^{1-\eta}\right]^{1 / \eta} .
\end{aligned}
$$

Under a cash platform, the consumer's solution has two regimes.

(a). If $\tau_{t} \geq \tau_{C}(i)$, then $q_{t}=m_{t}=\left[1-\tau_{C}(i)\right]^{1 / \eta-1}$, and $y_{t}=\left(1-\tau_{t}\right)^{1 / \eta} \leq\left(1-\tau_{t}\right) m_{t}$, where equality holds when $\tau_{t}=\tau_{C}(i)$;

(b). if $\tau_{t} \leq \tau_{C}(i)$, then $q_{t}=m_{t}=m\left(\tau_{t}\right)$, and $y_{t}=\left(1-\tau_{t}\right) m_{t}$.

The proof is provided in the Appendix. When $\tau$ is sufficiently high, the consumer's on-platform CIA constraint is not binding (case i). Inflation reduces off-platform transactions but has no effects on on-platform transactions. Transaction fees lower on-platform transactions but have no effects on off-platform transactions. When $\tau$ is sufficiently low, both CIA constraints are binding (case ii). Inflation and transaction fees reduce consumption on both platforms. Note that case (ii) is

\footnotetext{
${ }^{11}$ See Wong (2016) for the details of derivation.
} 
more likely when $i$ is high because $\tau_{C}(i)$ is an increasing function. The idea is that the consumer economizes on cash holdings when inflation is high, implying that the CIA constraint is more likely to be binding on the platform.

\subsection{Platform's problem}

The previous results suggest that, in setting the transaction fee, the platform has to take into account not only the negative impacts on platform consumption $y_{t}$ given the money holding $m_{t}$, but also the potential effects on the consumer's choice of $m_{t}$, which happens only in case (ii). The platform maximizes the discounted sum of profit flow given the consumer's solution:

$$
\Pi^{C} \equiv \max _{\tau_{t}} \sum_{t=1}^{\infty}(1-\gamma)^{t-1} \beta^{t} \alpha \sigma \tau_{t} \frac{\phi_{t} P_{t} y_{t}}{1-\tau_{t}}
$$

There is a measure $\alpha \sigma$ of consumers trading on the platform. Each consumer pays $\phi_{t} P_{t} y_{t} /\left(1-\tau_{t}\right)$ units of real balances of which the platform collects a fraction of $\tau_{t}$. Notice that (7) is a repeated static problem so its solution is time invariant. Using the two regimes of the consumer solution characterized by Lemma 1, we can rewrite the repeated static problem as

$$
\begin{aligned}
& \Pi^{C}=\frac{\alpha \sigma}{r+\gamma} \max \left\{\Pi_{1}^{C}, \Pi_{2}^{C}\right\}, \text { where } \\
& \Pi_{1}^{C} \equiv \max _{\tau_{t}} G_{1}\left(\tau_{t}\right) \text { s.t. } \tau_{t} \geq \tau_{C}(i) \\
& \Pi_{2}^{C} \equiv \max _{\tau_{t}} G_{2}\left(\tau_{t}\right) \text { s.t. } \tau_{t} \leq \tau_{C}(i) ;
\end{aligned}
$$

where $G_{1}(\tau) \equiv \tau(1-\tau)^{1 / \eta-1}$ and $G_{2}(\tau) \equiv \tau m(\tau)$. Here, the $\Pi_{1}^{C}$ function corresponds to case (i) in Lemma 1, where $\tau$ is sufficiently high to make the CIA constraint non-binding on the platform. Similarly, the $\Pi_{2}^{C}$ function corresponds to case (ii), where $\tau$ is sufficiently low to make the CIA constraint binding. In setting the optimal fee, the platform compares the values in the unconstrained and the constrained regions, $\Pi_{1}^{C}$ and $\Pi_{2}^{C}$, respectively.

Given these results, we can now derive the optimal fee $\tau$ chosen by the cash platform and the equilibrium quantities $(q, y)$ and platform profits $\Pi^{C}$, summarized in Proposition 1.

\section{Proposition 1 Define}

$$
\begin{aligned}
i^{*} & \equiv \sigma(1-\alpha)\left[(1-\eta)^{-(1-\eta)}-1\right] \\
\hat{\imath} & \equiv \sigma(1-\alpha)\left[(1-\bar{\tau})^{-(1-\eta)}-1\right]>i^{*} \\
\bar{\tau} & \equiv \arg \max \left\{\tau_{t}\left[\alpha\left(1-\tau_{t}\right)^{1-\eta}+(1-\alpha)\right]^{\frac{1}{\eta}}\right\} \in(\eta, 1) .
\end{aligned}
$$

Under a cash platform, the platform's solution has three regimes: 


\begin{tabular}{lcccc}
\hline \hline & $\tau_{t}$ & $q_{t}$ & $y_{t}$ & $\Pi^{C}$ \\
\hline (a). $i<i^{*}$ & $\eta$ & {$\left[1-\tau_{C}(i)\right]^{1 / \eta-1}$} & $(1-\eta)^{1 / \eta}$ & $\frac{\alpha \sigma}{r+\gamma} G_{1}(\eta)$ \\
(b). $i \in\left[i^{*}, \hat{\imath}\right]$ & $\tau_{C}(i)$ & {$\left[1-\tau_{C}(i)\right]^{1 / \eta-1}$} & {$\left[1-\tau_{C}(i)\right]^{1 / \eta}$} & $\frac{\alpha \sigma}{r+\gamma} G_{1}\left[\tau_{C}(i)\right]$ \\
(c). $i>\hat{\imath}$ & $\bar{\tau}$ & $m(\bar{\tau})$ & $(1-\bar{\tau}) q_{t}$ & $\frac{\alpha \sigma}{r+\gamma} G_{2}(\bar{\tau} ; i)$ \\
\hline \hline
\end{tabular}

The optimal fee is $\tau=\eta$ when the constraint (3) is non-binding, while $\bar{\tau}>\eta$ is the optimal fee when the constraint is binding. When the CIA constraint is non-binding on the platform, it is optimal to set a lower fee to maximize fee revenue. Setting $\tau=\eta$ is not feasible when the CIA constraint is binding, and the platform needs to raise $\tau$ to $\bar{\tau}$.

There are three cases. In case (a), as $i<i^{*}$, consumers hold sufficiently high cash balances so that the platform can set the fee at the unconstrained level $\tau=\eta$. The on-platform consumption $y$ is not affected by inflation, while the off-platform consumption $q$ and money holding $m$ are not affected by the fee rate.

As $i$ rises and enters the range $\left[i^{*}, \hat{\imath}\right]$ in case (b), the CIA starts to bind on the platform. The platform optimally raises the fee to $\tau=\tau_{C}(i) \in[\eta, \bar{\tau}]$, which induces a level of platform spending such that the CIA constraint is just binding. In this case, the marginal valuation of cash is not determined by platform activities. The on-platform consumption $y$ decreases with inflation, while the off-platform consumption $q$ and money holding $m$ are still independent of the fee rate.

When $i>\hat{\imath}$ in case (c), the consumers hold so little cash that the platform chooses to set a high $\tau=\bar{\tau}$, which implies the CIA is binding on the platform. In this case, the marginal valuation of cash is determined by platform activities. The fee rate and the inflation rate affect the money balances, the on-platform and the off-platform consumption.

To finish the study of the cash platform, we report some comparative statics in the following proposition.

Proposition 2 Under a cash platform, the comparative statics are given by

\begin{tabular}{lcccccccccc}
\hline \hline & $\frac{\partial \tau_{t}}{\partial i}$ & $\frac{\partial q_{t}}{\partial i}$ & $\frac{\partial y_{t}}{\partial i}$ & $\frac{\partial \Pi^{C}}{\partial i}$ & $\frac{\partial \tau_{t}}{\partial \alpha}$ & $\frac{\partial q_{t}}{\partial \alpha}$ & $\frac{\partial y_{t}}{\partial \alpha}$ & $\frac{\partial \Pi^{C}}{\partial \alpha}$ & $\frac{\partial \tau_{t}}{\partial \gamma}, \frac{\partial q_{t}}{\partial \gamma}, \frac{\partial y_{t}}{\partial \gamma}$ & $\frac{\partial \Pi^{C}}{\partial \gamma}$ \\
\hline (a). $i<i^{*}$ & 0 & - & 0 & 0 & 0 & - & 0 & + & 0 & - \\
(b). $i \in\left[i^{*}, \hat{\imath}\right]$ & + & - & - & - & + & - & - & \pm & 0 & - \\
(c). $i>\hat{\imath}$ & 0 & - & - & - & - & \pm & \pm & \pm & 0 & - \\
\hline \hline
\end{tabular}

Inflation generally has a negative effect on consumption and platform profits, with the exception of case (a), where on-platform activities are unconstrained by cash holdings. The effects of market share $\alpha$ is more complicated. In case (a), $\alpha$ reduces the off-platform marginal value of cash, while the on-platform marginal value of cash is not affected. This implies a lower $q$ but a fixed $y$. In case (b), the platform needs to raise $\tau$ to $\tau_{C}$, which increases with $\alpha$. Hence, the on-platform consumption $y$ declines with $\alpha$, too. In case (c), the platform raises the fee to $\bar{\tau}$. There are two opposite forces with respect to $\alpha$. First, the negative impact of the fee on cash holdings increases 
with $\alpha$. Second, the optimal fee $\bar{\tau}$ decreases with $\alpha$. As a result, the overall effects on quantities and profits are ambiguous. The effects of the resiliency parameter $\gamma$ are also intuitive. It reduces the (discounted) profits of the platform but has no effects on the fee rate or the quantities because the platform has a repeated static problem.

\section{Token platform}

We now consider a different business model in which the platform issues private IOUs, called tokens, in stage 2. In stage 1, consumers can then use these tokens to buy goods from merchants as the only acceptable medium of exchange on the platform. A token platform can make profits from charging transaction fees as well as selling new tokens. Issuing tokens incurs a setup cost $\kappa \alpha$, which is increasing in the market share $\alpha$ of platform trades. This captures the expenses of arranging the initial coin offering, building the new payment infrastructure and adhering to regulations. Define $p_{t}$ as the real price of tokens (in numeraire) if the cyber shock does not hit the platform. The nominal exchange rate of tokens is simply $S_{t} \equiv p_{t} / \phi_{t}$ (the amount of cash traded for each unit of tokens).

Notice that, unlike cash, the platform can fix the price of tokens $p_{t}$. How? One simple implementation is that the platform announces a price $p_{t}$ and stands ready to trade (redeem and issue) tokens in stage 2 at this targeted price. Tokens can keep circulating without redemption, which could happen when the redemption price is set below the stage 2 market price. Obviously, in an equilibrium with positive redemption, the market price is equal to the redemption price. ${ }^{12}$

Recall that the cyber shock hits with probability $\gamma$ in stage 2 . When this happens, the token can no longer be used for future platform transactions. A merchant who has accepted the tokens suffers the loss as the payment becomes worthless. Hence, the exchange value of tokens in stage 1 needs to price in the cyber risk in stage 2. To mitigate the token holder's exposure to the cyber risk, the platform can hold cash reserves to finance token redemption in case the platform collapses. Specifically, bearers can redeem each token for $B_{t}$ units of cash in stage 2 so that $b_{t} \equiv B_{t} / S_{t}$ is the real redemption rate. If $b_{t}=1$, then the token is fully backed and hence risk-free. To deliver on this promise, the platform issuing $K_{t}$ units of tokens needs to hold $B_{t} K_{t}$ units of cash reserves.

Before formally analyzing the token platform, we can briefly compare it to a cash platform to highlight some differences. Under a cash platform, inflation reduces platform transactions and profits whenever the CIA is binding on the platform. Using tokens to facilitate platform transactions can avoid the inflation tax. There are, however, costs associated with the token platform. First, issuing tokens incurs a setup cost. Second, token returns are risky due to the cyber risk, while cash is always safe. Third, the platform is subject to the opportunity cost of holding cash reserves to back the redemption price.

We now solve the equilibrium under a token platform. The merchant's problem is the same as that on the cash platform. To buy a unit of retail goods, the consumer needs to pay $P_{t}=1 / \phi_{t}$ units

\footnotetext{
${ }^{12}$ An alternative way, which does not involve actively trading with consumers, is that the platform can pay token holders an interest rate in terms of tokens. See Section 4.3 for details.
} 
of cash. Similarly, to buy retail goods using tokens, a consumer needs to pay $P_{t} /\left[\left(1-\gamma+\gamma b_{t}\right) S_{t}\right]$ units of tokens, where the denominator captures the associated risk and the exchange rate. We can then define $P_{t}^{T}=P_{t} /\left(1-\gamma+\gamma b_{t}\right)$ as the cash price of goods for a token user.

\subsection{Consumer's problem}

At the beginning of stage 1 in period $t$, the value function of a consumer holding $M$ units of cash and $K$ units of tokens is

$$
\begin{gathered}
V_{t}(M, K)=\max _{q_{t}, y_{t}}\left\{\begin{array}{c}
\sigma \alpha\left\{U\left(y_{t}\right)+W_{t}\left(M+S_{t} K-\frac{P_{t}^{T} y_{t}}{1-\tau_{t}}\right)\right\} \\
+\sigma(1-\alpha)\left[U\left(q_{t}\right)+W_{t}\left(M-P_{t} q_{t}+S_{t} K\right)\right] \\
+(1-\sigma) W_{t}\left(M+S_{t} K\right)
\end{array}\right\} \text { s.t. } \\
S_{t} K \geq \frac{P_{t}^{T} y_{t}}{1-\tau_{t}} \\
M \geq P_{t} q_{t}
\end{gathered}
$$

where $y_{t}$ is the quantity of retail goods bought on the token platform. In stage 1 , the nominal value of the tokens held by the consumer is $S_{t} K$. The token-in-advance constraint is given by (9). With probability $\sigma \alpha$, the consumer pays on the token platform $P_{t}^{T} y_{t} /\left(1-\tau_{t}\right)$ units of tokens for $y_{t}$ units of retail goods, of which $P_{t}^{T} y_{t}$ is paid to the merchant and $\tau_{t} P_{t}^{T} y_{t} /\left(1-\tau_{t}\right)$ to the platform as the transaction fees. The expected nominal value of balances (cash and tokens) brought to stage 2 is $M+S_{t} K-P_{t}^{T} y_{t} /\left(1-\tau_{t}\right)$.

At the beginning of stage 2 in period $t-1$, the value function of a consumer holding $A$ units of nominal balances (cash and tokens) is

$$
\begin{gathered}
W_{t-1}(A)=\max _{\left\{c_{t-1}, h_{t-1}, M_{t}, K_{t}\right\} \in \mathbb{R}_{+}^{4}}\left\{u\left(c_{t-1}, h_{t-1}\right)+\beta V_{t}\left(M_{t}, K_{t}\right)\right\}, \text { s.t. } \\
c_{t-1}+\phi_{t-1} M_{t}+p_{t-1} K_{t}=h_{t-1}+\phi_{t-1} A+\Upsilon_{t} .
\end{gathered}
$$

Applying the envelope theorem to (11) and (8), we obtain

$$
\begin{aligned}
& \frac{\partial V_{t}(M, K)}{\partial M}=\phi_{t}\left[1+\sigma(1-\alpha)\left\{U^{\prime}\left(m_{t}\right)-1\right\}_{+}\right], \\
& \frac{\partial V_{t}(M, K)}{\partial K}=p_{t}\left(1-\gamma+\gamma b_{t}\right)\left[1+\sigma \alpha\left\{\left(1-\tau_{t}\right) U^{\prime}\left[\left(1-\tau_{t}\right) k_{t}\right]-1\right\}_{+}\right],
\end{aligned}
$$

where $k_{t} \equiv\left[1-\gamma\left(1-b_{t}\right)\right] p_{t} K_{t}$ denotes the real token balances. When $b_{t}=1$, the full real value of tokens is always guaranteed. The first-order conditions of (11) with respect to $M_{t}$ and $K_{t}$ are

$$
\begin{aligned}
i & =\sigma(1-\alpha)\left\{m_{t}^{-\eta}-1\right\}_{+}, \\
\rho_{t} & =\sigma \alpha\left\{\left(1-\tau_{t}\right)^{1-\eta} k_{t}^{-\eta}-1\right\}_{+}
\end{aligned}
$$


where

$$
\rho_{t} \equiv \frac{(1+r) p_{t-1}}{p_{t}\left[1-\gamma\left(1-b_{t}\right)\right]}-1
$$

is the token yield - the interest rate needed to compensate the token holder for bearing the cyber risk, discounting, and changes in token prices. The RHS of (13) is the marginal value of holding tokens, consisting of the liquidity value of using tokens on the platform. Under a token platform, the consumer's solution is given by

$$
\begin{aligned}
& q_{t}=m_{t}=\left[1+\frac{i}{\sigma(1-\alpha)}\right]^{-1 / \eta}, \\
& y_{t}=\left(1-\tau_{t}\right)^{1 / \eta}\left(1+\frac{\rho_{t}}{\sigma \alpha}\right)^{-1 / \eta}, \\
& k_{t}=\left\{\begin{array}{l}
\left(1-\tau_{t}\right)^{1 / \eta-1}\left(1+\frac{\rho_{t}}{\sigma \alpha}\right)^{-1 / \eta}, \text { if } \rho_{t}>0, \\
\in\left[\left(1-\tau_{t}\right)^{1 / \eta-1}, \infty\right], \text { if } \rho_{t}=0 .
\end{array}\right.
\end{aligned}
$$

Intuitively, the on-platform consumption decreases with $\rho, \gamma, \tau$ and increases with $b$. Finally, note that the aggregate stage- 2 utility is a constant and equal to $w_{t}$ for all $t$, similar to the case of the cash platform.

\subsection{Platform's problem}

The initial profit flow of the platform in stage 2 is

$$
x_{0}=p_{0} K_{1}-\kappa \alpha-\bar{\mu} b_{1} p_{1} K_{1}
$$

which consists of the revenue from the initial issuance of $K_{1}$ units of tokens each worth $p_{0}$ units of numeraire net of the setup cost and the amount of cash reserves the platform holds to back up its redemption in the following period. Each token is redeemable for $B_{1}=b_{1} p_{1}$ units of cash in period 1 , and each unit of period-1 cash is worth $\bar{\mu}=\phi_{0} / \phi_{1}$ units of numeraire in period 0 . The expected profit flow in any subsequent period is

$$
\begin{aligned}
x_{1 t} & =p_{t}\left(b_{t} K_{t}+\frac{K_{t+1}}{1+i}-K_{t}-\frac{\bar{\mu}}{p_{t}} b_{t+1} p_{t+1} K_{t+1}\right)+\sigma \alpha \tau_{t} \frac{\phi_{t} P_{t}^{T} y_{t}}{1-\tau_{t}}, \\
x_{0 t} & =\sigma \alpha \tau_{t} b_{t} \frac{\phi_{t} P_{t}^{T} y_{t}}{1-\tau_{t}} .
\end{aligned}
$$

If the cyber shock does not hit in stage 2, then the profit flow consists of the reserves brought forward $\left(p_{t} b_{t} K_{t}\right)$, the sale of new tokens, $\left[p_{t}\left(K_{t+1}-K_{t}\right)\right]$, the receipt of transaction fees in tokens $\left[\sigma \alpha \tau_{t} \phi_{t} P_{t}^{T} y_{t} /\left(1-\tau_{t}\right)\right]$, subtracting the expenses on new cash reserves $\left(\bar{\mu} b_{t+1} p_{t+1} K_{t+1}\right)$. If the cyber shock hits in stage 2, then token holders redeem the platform's cash reserves, leaving an amount

$\sigma \alpha \tau_{t} b_{t} \phi_{t} P_{t}^{T} y_{t} /\left(1-\tau_{t}\right)$ to the platform (which captures the reserves held to back the fee revenue received by the platform). 
The platform's profit is given by $\Pi^{T}=\max \left\{x_{0}+\sum_{t=1}^{\infty}(1-\gamma)^{t-1} \beta^{t}\left[(1-\gamma) x_{1 t}+\gamma x_{0 t}\right]\right\}$. By collecting terms with the same $t$ across periods, we can express the platform's problem as

$$
\Pi^{T}=-\kappa \alpha+\max _{b_{t}, k_{t}, \tau_{t} \geq 0}\left\{\sum_{t=1}^{\infty}(1-\gamma)^{t-1} \beta^{t}\left[\rho_{t} k_{t}+\sigma \alpha\left[1-\gamma\left(1-b_{t}\right)\right] \frac{\tau_{t} y_{t}}{1-\tau_{t}}-\frac{i b_{t}}{1-\gamma\left(1-b_{t}\right)} k_{t}\right]\right\} .
$$

The optimal design of a token platform is summarized by the following proposition.

Proposition 3 Under a token platform, the solution of the platform's problem is given by

$$
\begin{aligned}
\tau_{t} & \in[0, \eta], b_{t}=0, \rho_{t}=\frac{\sigma \alpha}{1-\eta}\left(\eta-\tau_{t}\right), \\
q_{t} & =\left[1-\tau_{C}(i)\right]^{1 / \eta-1} \\
y_{t} & =(1-\eta)^{1 / \eta} \\
\Pi^{T} & =-\kappa \alpha+\frac{\sigma \alpha \eta}{r+\gamma}(1-\eta)^{1 / \eta-1} .
\end{aligned}
$$

The platform monopoly designs the features $\left\{\rho_{t}, \tau_{t}, b_{t}\right\}$ to extract the consumer's surplus of the platform trades. Rearranging terms, the expected profit flow in $t$ is proportional to

$$
\sigma \alpha\left(y_{t}^{-\eta}-1\right) y_{t}-\frac{i b_{t}}{1-\gamma\left(1-b_{t}\right)} k_{t}
$$

In this economy, it is the purchasing power of tokens, $y_{t}=\left(1-\tau_{t}\right) k_{t}$, that matters for the consumer surplus, which is proportional to the first term above, $\left(y_{t}^{-\eta}-1\right) \sigma \alpha y_{t}$, as the marginal utility of retail consumption net of real price (which is unity) times the total retail consumption purchased by the tokens. The platform can implement any $y_{t}$ by varying the reserve $b_{t}$, the fee $\tau$, and the token interest rate $\rho_{t}$, via the pricing kernel (13). Increasing reserves holding $b_{t}$ helps support the purchasing power of tokens, albeit raising the opportunity costs $i b_{t} k_{t}$ in terms of the interest forgone. A less costly way to support the purchasing power of tokens without this opportunity cost of the interest forgone is to reduce $\tau_{t}$ and $\rho_{t}$. Thus, it is optimal not to back tokens by any reserves. Finally, the token is indifferent to a range of $\rho_{t}$ and $\tau_{t}$ because charging a higher transaction fee can always be replicated by selling tokens at a higher price without the transaction fee.

We first define the growth rate of the nominal token price as $g_{t} \equiv S_{t} / S_{t-1}$. The following proposition reports some comparative statics results.

Proposition 4 Under a token platform, fixing $\tau_{t} \in[0, \eta)$, the comparative statics are given by

\begin{tabular}{lllll}
\hline \hline & $\partial g_{t}$ & $\partial q_{t}$ & $\partial y_{t}$ & $\partial \Pi^{T}$ \\
\hline$\partial i$ & + & - & 0 & 0 \\
$\partial \alpha$ & - & - & 0 & + \\
$\partial \gamma$ & + & 0 & 0 & - \\
\hline \hline
\end{tabular}


Propositions 1 and 2 suggest that, under a cash platform, platform consumption $y$ and profits $\Pi^{C}$ are both maximized when $i<i^{*}$. Higher $i$ tightens the CIA constraint on the platform and reduces platform transactions and profits. By issuing tokens, the platform can replicate the maximum consumption and profits under a cash platform as the usage of tokens helps avoid inflation tax. The platform can maintain a fixed consumption level $y$ by adjusting the nominal price paths of tokens (through $g_{t}$ ) in response to changes in $i, \alpha$, and $\gamma$. Interestingly, while a higher $\gamma$ increases the risk of holding tokens, the platform can simply compensate users by appreciating token prices faster without the need to hold costly cash reserves.

\subsection{Alternative implementation: interest-bearing stablecoins}

An alternative way to implement the token solution is to pay interest in terms of tokens. One realworld example is the interesting-bearing Yu'ebao accounts offered by Alipay. Here, tokens become a "digital asset" working like a money market mutual fund. As we will show, by administering the interest rates, the platform can ensure that tokens are traded at par with cash, like stablecoins.

Suppose the platform pays an interest of $\nu_{t}$ (in terms of of tokens) for each unit of tokens held in the beginning of stage 2. By modifying the definition to $k_{t} \equiv\left[1-\gamma\left(1-b_{t}\right)\right]\left(1+\nu_{t}\right) p_{t} K_{t}$, we obtain the following first-order condition with respect to $K_{t}$ :

$$
\frac{(1+r) p_{t-1}}{p_{t}\left(1+v_{t}\right)\left[1-\gamma\left(1-b_{t}\right)\right]}-1=\sigma \alpha\left\{\left(1-\tau_{t}\right)^{1-\eta} k_{t}^{-\eta}-1\right\}_{+} .
$$

The optimal allocation of Proposition (4) can be implemented under $p_{t}=\phi_{t}$, by setting

$$
v_{t}=\frac{1+i}{\left(1+\rho_{t}\right)(1-\gamma)}-1
$$

where $\rho_{t}=\sigma \alpha \eta /(1-\eta), b_{t}=0$ and $\tau_{t}=0$. In this case, the platform issues stablecoins which pay interests at a rate below the cyber-risk adjusted nominal interest rate.

\subsection{Digression: acceptance of cash on token platforms}

We have implicitly assumed that a token platform does not accept cash. We here consider the general setting and show that accepting both cash and tokens is not optimal. Suppose the platform has an additional option of running a dual payments business model where both cash and tokens can be used on the platform. The consumer's problem becomes

$$
V_{t}(M, K)=\max _{q_{t}, y_{t}^{M} \geq 0, y_{t} \geq 0}\left\{\begin{array}{l}
\sigma(1-\alpha)\left\{U\left(q_{t}\right)+W_{t}\left(M+S_{t} K-P_{t} q_{t}\right)\right\} \\
+\sigma \alpha\left[U\left(y_{t}^{M}+y_{t}^{K}\right)+W_{t}\left(M-\frac{P_{t} y_{t}^{M}}{1-\tau_{t}^{M}}+S_{t} K-\frac{P_{t}^{T} y_{t}}{1-\tau_{t}}\right)\right] \\
+(1-\sigma) W_{t}\left(M+S_{t} K\right)
\end{array}\right\} \text { s.t. }
$$




$$
\begin{aligned}
M & \geq P_{t} q_{t}, \\
M & \geq \frac{P_{t} y_{t}^{M}}{1-\tau_{t}^{M}} \\
S_{t} K & \geq \frac{P_{t}^{T} y_{t}^{K}}{1-\tau_{t}^{K}},
\end{aligned}
$$

where $q_{t}$ denotes off-platform consumption paid with cash, $y_{t}^{M}$ denotes on-platform consumption paid with cash, and $y_{t}^{K}$ denotes on-platform consumption paid with tokens. Note that all onplatform transactions are subject to transaction fees: $\tau_{t}^{M}$ for cash and $\tau_{t}^{K}$ for token.

We focus on the case where the consumer's token holding is positive, otherwise, it is just a de facto cash platform. This means the token-in-advance constraint (19) is binding, i.e., $y_{t}^{K}=$ $\left(1-\tau_{t}^{K}\right) k_{t}$. The shadow rate of tokens is given by the first order condition w.r.t. $k_{t}$ :

$$
\begin{aligned}
\rho_{t} & =\sigma \alpha\left[\left(1-\tau_{t}^{K}\right) y_{t}^{-\eta}-1\right] \\
i & =\sigma(1-\alpha)\left\{m_{t}^{-\eta}-1\right\}_{+}+\sigma \alpha\left\{\left(1-\tau_{t}^{M}\right) y_{t}^{-\eta}-1\right\}_{+}
\end{aligned}
$$

where $y_{t}=y_{t}^{M}+y_{t}^{K}$. We also focus on the case that cash is used on the platform (otherwise it is just a de facto token platform). Since cash and tokens are perfect substitutes, cash usage on the platform is "bang-bang", i.e., either $y_{t}^{M}=\left(1-\tau_{t}^{M}\right) m_{t}$ or $y_{t}^{M}=0$. The consumer uses all the cash she holds only if

$$
\left(1-\tau_{t}^{M}\right) y_{t}^{-\eta} \geq 1
$$

Here, the marginal benefit of using cash balances on platform consumption is $\left(1-\tau_{t}^{M}\right) y_{t}^{-\eta}$, and the marginal cost is one (the forgone opportunity of buying numeraires with cash in stage 2 ). The platform problem becomes

$$
\begin{aligned}
\Pi^{D}= & -\kappa \alpha+\max \left\{\sum_{t=1}^{\infty}(1-\gamma)^{t-1} \beta^{t}\left\{\rho_{t} k_{t}+\sigma \alpha \tau_{t}^{K} k_{t}+\sigma \alpha \tau_{t}^{M} m_{t}-\frac{i b_{t}}{1-\gamma\left(1-b_{t}\right)} k_{t}\right\}\right\} \\
& \text { s.t. (21), (22) and (23). }
\end{aligned}
$$

Proposition 5 A dual platform is always dominated by a cash or a token platform in terms of profits, i.e., $\Pi^{D} \leq \max \left\{\Pi^{C}, \Pi^{T}\right\}$.

This result helps us understand why blockchain-based platforms issue utility tokens as the only means of payments. At the same time, there do exist dual systems where both tokens and cash are accepted on the platform. The model can potentially be extended to generate this as an equilibrium outcome. For example, if consumers are heterogeneous in terms of their costs of adopting tokens, then the platform might choose to adopt a dual system where cash is used by high adoption cost consumers (e.g. the elderly) and tokens used by others. We leave this for future research. 


\section{$5 \quad$ Equilibrium business model and welfare}

We now study the platform's choice to accept cash or issue tokens and the welfare implications of that choice.

\subsection{Platform's business model choice}

Obviously, the platform chooses to issue tokens, instead of using cash, whenever $\Pi^{T}>\Pi^{C}$. The three key parameters are $i, \gamma$, and $\alpha$. Lemma 2 completely characterizes the parameter space such that the platform issues tokens instead of using cash.

Lemma 2 There exists a threshold interest rate $\iota^{\Pi}(\alpha, \gamma)>i^{*}$ such that $\Pi^{T}>\Pi^{C}$ if and only if $i>\iota^{\Pi}(\alpha, \gamma)$. Moreover, $\iota^{\Pi}(\alpha, \gamma)$ is a function decreasing in $\alpha$ but increasing in $\gamma$.

As discussed above, when $i<i^{*}$, the tax collected on the cash platform is the same as the token sale revenue collected from the token platform because the CIA constraint is not binding on the cash platform. Hence, the token platform is dominated due to the additional cost. As $i$ increases, the CIA constraint on the cash platform starts to bind. The token platform dominates for high $i$ because the usage of tokens insulates the platform from the inflation tax.

The token platform dominates the cash platform when the share of platform trade $(\alpha)$ is sufficiently high. Two channels explain why this happens. First, a higher $\alpha$ increases the extensive margin of platform trades, which raises the revenues of both the token and cash platform proportionally. Second, a higher $\alpha$ will have potential effects on the intensive margin of platform trades. A higher $\alpha$ means there is less off-platform trade, implying a lower cash holding. When the CIA constraint is not binding, the fee revenue per trade is unaffected. When the CIA constraint is binding, the fee revenue per trade falls in proportion to the cash holding. To counteract that effect, the cash platform reduces $\tau_{t}$. The overall effect of $\alpha$ is thus ambiguous on a cash platform. However, a higher $\alpha$ does not change the intensive margin under a token platform because it does not alter the consumer surplus per platform trade. Hence, the token platform does not want to change the distortion. In sum, when $\alpha$ increases, the profit increases more under a token model than a cash model. When the premium in profit justifies the fixed cost of issuing tokens, the token platform dominates. Finally, as the platform becomes less resilient (higher $\gamma$ ), the platform is less willing to issue tokens because $\gamma$ reduces the expected returns to pay a setup cost to start a token platform.

We now consider the general-equilibrium effects of the platform's decision to issue tokens. First, to examine the effects on trading activities, we define the off-platform social surplus as $U(q)-q$ and the on-platform social surplus as $U(y)-y$. To study how the platform's business model affects the central bank's seigniorage revenue, we define the equilibrium seigniorage from cash issuance as

$$
\begin{aligned}
\mathcal{S} & =\phi_{0} M_{1}+\bar{\mu} b_{1} p_{1} K_{1}+\sum_{t=1}(1-\gamma)^{t-1} \beta^{t}\left\{\begin{array}{l}
(1-\gamma)\left[\phi_{t}\left(M_{t+1}-M_{t}\right)-p_{t} b_{t} K_{t}+\bar{\mu} b_{t+1} p_{t+1} K_{t+1}\right] \\
\gamma\left(-p_{t} b_{t} K_{t}+\overline{\mathcal{S}}\right)
\end{array}\right\} \\
& =\frac{i+\gamma}{r+\gamma} m+\frac{i}{r+\gamma} \frac{b}{1-\gamma(1-b)} k+\frac{\gamma}{r+\gamma} \overline{\mathcal{S}}
\end{aligned}
$$


where $\overline{\mathcal{S}}$ is the continuation present value of the seigniorage revenue after the cyber shock hits. We can use the above findings to obtain the following proposition.

Proposition 6 When the platform chooses to issue tokens instead of accepting cash,

1. on-platform consumption and social surplus go up,

2. off-platform consumption and social surplus go down,

3. seigniorage revenue goes down.

When the platform issues tokens instead of accepting cash, it insulates the platform transactions from the inflation tax. This will not only increase profits but also increase the total trade surplus on the platform. However, this has a spillover effect on off-platform transactions. The switch to a token platform lowers the demand for cash, and hence the off-platform transactions and surplus go down as the CIA constraint becomes more binding. Finally, since the token platform does not hold cash reserves, the central bank's seigniorage revenue will decline too.

\subsection{Welfare implications}

We now investigate whether the business model chosen by the platform also maximizes social welfare. The welfare of this economy is defined as the discounted sum of utilities of consumers, merchants and the platform, which are given by

$$
\begin{aligned}
& \mathcal{W}^{C}=\frac{1}{r+\gamma}\left\{\sigma \alpha\left[U\left(y^{C}\right)-y^{C}\right]+\sigma(1-\alpha)\left[U\left(q^{C}\right)-q^{C}\right]+w_{t}\right\}+\frac{\gamma \beta}{r+\gamma} \overline{\mathcal{W}} \\
& \mathcal{W}^{T}=-\kappa \alpha+\frac{1}{r+\gamma}\left\{\sigma \alpha\left[U\left(y^{T}\right)-y^{T}\right]+\sigma(1-\alpha)\left[U\left(q^{T}\right)-q^{T}\right]+w_{t}\right\}+\frac{\gamma \beta}{r+\gamma} \overline{\mathcal{W}},
\end{aligned}
$$

where $\mathcal{W}^{C}$ is the welfare under a cash platform, $\mathcal{W}^{T}$ is the welfare under a token platform, and $\overline{\mathcal{W}}$ is the continuation welfare after the cyber shock. The following lemma describes when issuing tokens maximizes social welfare.

Lemma 3 There exists a threshold rate $\iota^{\mathcal{W}}(\alpha, \gamma)>i^{*}$ such that $\mathcal{W}^{T}<\mathcal{W}^{C}$ if and only if $i<$ $\iota^{\mathcal{W}}(\alpha, \gamma)$. Moreover, $\iota^{\mathcal{W}}(\alpha, \gamma)$ is a function decreasing in $\alpha$ if $i \leq \hat{\imath}$, but increasing in $\gamma$.

The effects of $i$ and $\gamma$ on welfare are similar to those on the platform's profits. When $i<$ $i^{*}$, a token platform and a cash platform support the same consumption allocations and profits. Therefore, the token platform is dominated due to the additional setup cost. As $i$ increases, both platform profits and consumption drop when a cash platform is chosen. Hence, a token platform is preferred when the inflation rate is sufficiently high because tokens insulate the platform consumption from inflation tax. Furthermore, an increase in $\gamma$ reduces the expected benefits of setting up a token platform. 


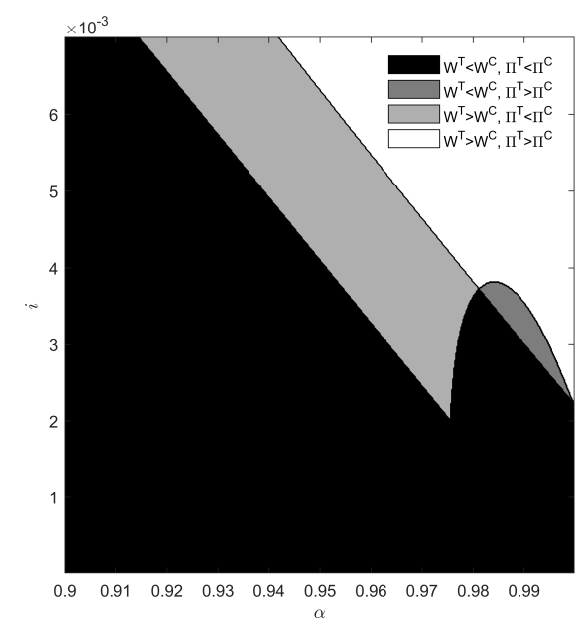

Figure 1: Profits vs welfare $(\gamma=0.8)$

The effects of $\alpha$ on welfare can be different from those on the platform's profits when $i>\hat{\imath}$. In particular, for $i>\hat{\imath}$, it could well be that $\mathcal{W}^{C}$ overtakes $\mathcal{W}^{T}$ when $\alpha$ becomes higher. This is due to the spillover effects on off-platform surplus discussed in the previous proposition. On the other hand, $\Pi^{C}$ never overtakes $\Pi^{T}$. This is because the cash demand decreases when $\alpha$ becomes higher. Since the cash platform relies on cash trades for revenue, it responds by cutting the transaction fee to mitigate the reduction in cash holding. Doing so reduces the distortion on the platform trades, thus the welfare under the cash platform can be higher than it is under the token platform.

The equilibrium choice of the platform is not socially optimal when the welfare could increase by making the alternative choice. This happens when $\Pi^{T}>\Pi^{C}$ but $\mathcal{W}^{T}<\mathcal{W}^{C}$ or when $\Pi^{T}<\Pi^{C}$ but $\mathcal{W}^{T}>\mathcal{W}^{C}$. In the former, a token platform is chosen in equilibrium but a cash platform yields higher welfare. By using Lemmas 2 and 3, the following corollary summarizes the welfare properties of the equilibrium business model.

Corollary 1 A cash platform is a socially optimal business model if and only if $i \leq \min \left\{\iota^{\Pi}(\alpha, \gamma), \iota^{\mathcal{W}}(\alpha, \gamma)\right\}$. The measure of this parameter subspace decreases with $\alpha$ if $i \leq \hat{\imath}$ but increases with $\gamma$. A token platform is a socially optimal business model if and only if $i \geq \max \left\{\iota^{\Pi}(\alpha, \gamma), \iota^{\mathcal{W}}(\alpha, \gamma)\right\}$. The measure of this parameter subspace increases with $\alpha$ if $i \leq \hat{\imath}$ but decreases with $\gamma$.

The equilibrium choice of business model can be suboptimal because the platform only considers its profits and does not internalize the effects on the consumers' surplus (merchants have zero surplus). In particular, a token platform is suboptimally adopted when $i \in\left(\iota^{\Pi}(\alpha, \gamma), \iota^{\mathcal{W}}(\alpha, \gamma)\right)$ and a cash platform is suboptimally adopted when $i \in\left(\iota^{\mathcal{W}}(\alpha, \gamma), \iota^{\Pi}(\alpha, \gamma)\right)$. A simple way to correct these suboptimal outcomes is to impose a tax $T$ on token platform adoption (subsidy when negative). The government can induce the platform to choose the socially optimal business model by imposing on the token platform a $\operatorname{tax} T=\left(\mathcal{W}^{C}-\Pi^{C}\right)-\left(\mathcal{W}^{T}-\Pi^{T}\right)$. 
Table 1: Welfare properties of platforms

\begin{tabular}{|c|c|c|c|c|c|c|c|}
\hline & \multicolumn{7}{|c|}{ Example 1: $\alpha=0.96, i=0.34, \gamma=0.8$} \\
\hline & \multirow{2}{*}{$\begin{array}{l}\text { off-platform } \\
\qquad q\end{array}$} & \multirow{2}{*}{$\begin{array}{c}\text { on-platform } \\
y\end{array}$} & \multirow{2}{*}{$\begin{array}{c}\text { welfare } \\
\qquad W\end{array}$} & \multirow{2}{*}{$\begin{array}{c}\text { profits } \\
\Pi\end{array}$} & \multicolumn{3}{|c|}{ buyers'surplus } \\
\hline & & & & & $\bar{U}^{q}$ & $\bar{U}^{y}$ & $\bar{U}$ \\
\hline Cash platform & 0.3674 & 0.3368 & 0.0755 & 0.0354 & 0.0031 & 0.0369 & 0.0401 \\
\hline Token platform & 0.3607 & 0.3527 & 0.0761 & 0.0345 & 0.0031 & 0.0385 & 0.0416 \\
\hline & & Example 2 & $\alpha=0.9$ & $i=0$. & $\gamma=0$ & & \\
\hline Cash platform & 0.3674 & 0.3377 & 0.0755 & 0.0354 & 0.0008 & 0.0393 & 0.0401 \\
\hline Token platform & 0.0258 & 0.3527 & 0.0754 & 0.0355 & 0.0001 & 0.0397 & 0.0399 \\
\hline
\end{tabular}

\subsection{Numerical examples}

We study a numerical example with the following parameter values: $\beta=0.97, \sigma=1, \eta=0.08$, $\kappa=0.001$. Figure 1 compares the relative ranking of a cash platform and a token platform in terms of social welfare and the platform's profits for different levels of interest rate $i$ and platform fragility $\alpha$. As implied by Corollary 1, the platform's choice of its business model (i.e., cash vs. token platforms) can be socially optimal or not. In particular, there are altogether four regions in the $(i, \alpha)$ parameter space, depending on whether the platform's choice and the social planner's choice are aligned or not. Note that the region where cash is optimal is hump-shaped for higher values of $\alpha$ due to the trade-off between the positive effect on on-platform surplus and the negative effect on off-platform social surplus.

To illustrate that the equilibrium business can be suboptimal, in Table 1, we decompose welfare into profits and consumers' surplus (denoted by $\bar{U}$ ) under the cash and token platforms. In the first example, the platform suboptimally chooses not to issue tokens. As shown in the upper panel of Table 1, switching from a cash platform to a token platform increases consumers' surplus, which is big enough to offset the platform's profit loss. In the second example, the platform suboptimally chooses to issue tokens. As shown in the lower panel of Table 1, switching from a token platform to a cash platform can significantly increase off-platform consumption and hence increase welfare.

To understand these findings, note that in choosing its business model, the platform considers only the effects on its profits:

$$
\Pi^{T}-\Pi^{C}=-\kappa \alpha+\frac{\sigma \alpha}{r+\gamma}\left(\frac{\eta}{1-\eta} y^{T}-\frac{\tau}{1-\tau} y^{C}\right) .
$$

However, the platform does not fully internalize the effects on the consumers' surplus, which is 
given by

$$
\frac{\sigma}{r+\gamma}\{\alpha \underbrace{\left[U\left(y^{T}\right)-U\left(y^{C}\right)-\frac{y^{T}}{1-\eta}+\frac{y^{C}}{1-\tau}\right]}_{\text {change in on-platform surplus } \bar{U}^{y}}+(1-\alpha) \underbrace{\left[U\left(q^{T}\right)-U\left(q^{C}\right)-q^{T}+q^{C}\right]}_{\text {change in off-platform surplus } \bar{U}^{q}}\} .
$$

This is a weighed sum of the effects on consumers' on-platform surplus $\bar{U}^{y}$ and off-platform surplus $\bar{U}^{q}$ with the weight given by $\alpha$. As $\alpha$ goes up, the on-platform surplus goes up, while the on-platform surplus goes down. In the first (second) example where $\alpha$ is lower (higher), the on-platform (offplatform) surplus change dominates. Since the platform fails to fully internalize the social benefits (costs) of issuing tokens in the first (second) example, the platform tends to under-issue (over-issue) tokens.

\section{Token platform regulation}

The above discussion concerns the optimality of adopting a token platform. Conditional on its adoption, the allocation under a token platform can still be suboptimal. Relative to the first-best, there is under-consumption of platform goods when the purchasing power of tokens is so low that $y_{t} \leq 1$. Recall that the purchasing power of tokens is $y_{t}=\left(1-\tau_{t}\right) k_{t}$. From (15), we know that the demand for tokens is low when the transaction fee is high, or the shadow rate is high (tokens depreciate quickly), or the cyber risk is high, or the reserve backing is low. Since the token platform charges the minimal transaction fee, $\tau_{t}=0$, the other three are the potential sources of welfare loss. A natural question is whether the government can impose certain policies to reduce welfare loss. In reality, widely circulated private currencies are often subject to a minimum reserve requirement (e.g., PBoC's regulation on Alipay and WeChat). We consider below whether regulating the reserve holding of the platform is welfare-improving. In the Appendix, we also study whether providing deposit insurance can reduce the impacts of the cyber risk.

Suppose the central bank introduces a minimum reserve requirement on the token platform such

that at least a fraction $\bar{b}$ of the token value is backed by cash reserves, i.e., $b_{t} \geq \bar{b}$. The platform problem becomes

$$
\Pi^{R}=-\kappa \alpha+\max _{k_{t}, \tau_{t} \geq 0}\left\{\sum_{t=1}^{\infty}(1-\gamma)^{t-1} \beta^{t}\left[\rho_{t}-i+(1-\gamma) \sigma \alpha \tau_{t}\right] k_{t}\right\} .
$$

The equilibrium quantities and profits are given by the following proposition. 
Proposition 7 Under a reserve requirement, the solution of a token platform is $b_{t}=\bar{b}, \tau_{t}=0$,

$$
\begin{aligned}
\rho_{t} & =\frac{1}{1-\eta}\left[\sigma \alpha \eta-\frac{i \bar{b}}{1-\gamma(1-\bar{b})}\right] \\
q_{t} & =\left[1-\tau_{C}(i)\right]^{1 / \eta-1} \\
y_{t} & =\left[\frac{1-\eta}{1+\frac{i}{\sigma \alpha} \frac{\bar{b}}{1-\gamma(1-\bar{b})}}\right]^{1 / \eta}, \\
\Pi^{R} & =-\kappa \alpha+\frac{\sigma \alpha \eta}{r+\gamma} y_{t}^{1-\eta}
\end{aligned}
$$

The token platform under a higher reserve requirement features lower trading activities $y_{t}$. This is not due to a higher transaction fee as the platform sets $\tau_{t}$ at 0 . Recall that from (15), the demand for tokens is lower if the reserve backing is lower or the shadow rate is higher. The reserve requirement increases cash reserves and actually increases the demand for tokens. However, the platform takes advantage of the higher demand by charging a far higher shadow rate $\rho$, which ultimately pushes down the token demand. But this does not mean the platform makes more profit because holding reserves is costly and the higher shadow rate is to compensate that. Overall, both the consumer surplus and platform profit fall after a higher reserve requirement. Hence, conditional on the usage of a token platform, a higher reserve requirement only reduces welfare. Nevertheless, a sufficiently high reserve requirement could improve welfare when the platform's choice of token issuance is suboptimal (see Corollary 1) because it induces the platform to use cash instead of issuing tokens. The following corollary summarizes these findings.

Corollary 2 The token platform under a higher reserve requirement features a lower $g_{t}$, a lower platform trade volume and a lower platform profit. Reserve requirement potentially results in a higher welfare only if the platform ceases to issue tokens.

To deal with the default risk of a deposit-taking institution, a commonly used regulation is deposit insurance. In the appendix, we study and show that the provision of deposit insurance does not affect welfare because its effect is offset one-to-one by the corresponding decrease in $g_{t}$, while the profits and allocation stay unchanged.

\section{$7 \quad$ Endogenous interoperability}

One concern about private digital currencies is that the lack of payment interoperability across platforms can increase the fragmentation of the payment system (Brunnermeier, James and Landau, 2019). We have already shown that a token platform has no incentives to accept cash. We will now examine whether the platform wants to promote the circulation of tokens outside the platform.

Suppose the platform has an option to circulate tokens and provide payment services outside the platform. We call these "circulatable tokens". In reality, private currencies that are widely 
accessible tend to be subject to regulation due to concerns about financial risk and consumer protection. For example, China's central bank requires payment firms such as Alipay and WeChat to deposit reserves of client funds with a centralized custodian. To capture this real-world regulation, we assume that the token platform has to satisfy a minimum reserve ratio $b \in[0,1]$. The platform cannot charge fees on transactions outside the platform. To simplify the analysis, we also assume $\tau_{t}=0$ on the platform. The consumer problem becomes

$$
\begin{gathered}
V_{t}(M, K)=\max _{q_{t}, y_{t}}\left\{\begin{array}{l}
\sigma \alpha\left\{U\left(y_{t}\right)+W_{t}\left(M+S_{t} K-P_{t}^{T} y_{t}\right)\right\} \\
+\sigma(1-\alpha)\left[U\left(q_{t}^{M}+q_{t}^{K}\right)+W_{t}\left(M-P_{t} q_{t}+S_{t} K-P_{t}^{T} q_{t}^{K}\right)\right] \\
+(1-\sigma) W_{t}\left(M+S_{t} K\right)
\end{array}\right\} \text { s.t. } \\
S_{t} K \geq P_{t}^{T} y_{t}, M \geq P_{t} q_{t}^{M}, S_{t} K \geq P_{t}^{T} q_{t}^{K} .
\end{gathered}
$$

The first order conditions are given by

$$
\begin{aligned}
i & \geq \sigma(1-\alpha)\left\{\left(m_{t}+k_{t}\right)^{-\eta}-1\right\}_{+}, \text {equal when } m_{t}>0 \\
\rho_{t} & =\sigma \alpha\left\{k_{t}^{-\eta}-1\right\}_{+}+\sigma(1-\alpha)\left\{\min \left\{k_{t}^{-\eta}, 1+\frac{i}{\sigma(1-\alpha)}\right\}-1\right\}_{+} .
\end{aligned}
$$

The consumer's solution is given by the following lemma.

Lemma 4 Under a circulatable token platform, the consumer's solution has two regimes:

\begin{tabular}{lcc}
\hline \hline & $q_{t}^{M}=m_{t}$ & $q_{t}^{K}=y_{t}=k_{t}$ \\
\hline (a). $i \leq(1-\alpha) \rho_{t}$ & {$\left[1+\frac{i}{\sigma(1-\alpha)}\right]^{-1 / \eta}-k_{t}$} & $\left(1+\frac{\rho_{t}-i}{\sigma \alpha}\right)^{-1 / \eta}$ \\
(b). $i>(1-\alpha) \rho_{t}$ & 0 & $\left(1+\frac{\rho_{t}}{\sigma}\right)^{-1 / \eta}$ \\
\hline \hline
\end{tabular}

Circulatable tokens and cash are in direct competition off-platform. When $i$ is sufficiently high (case ii), tokens can drive out cash and become the only payment instrument in the retail sector (even though there is still demand for cash as a reserve asset). When $i$ is not too high (case i), cash is still used off-platform.

The platform problem is

$$
\Pi^{C T}=-\kappa \alpha+\max _{b_{t} \geq b, k_{t}}\left\{\sum_{t=1}^{\infty}(1-\gamma)^{t-1} \beta^{t}\left[\rho_{t}-\frac{i b}{1-\gamma(1-b)}\right] k_{t}\right\} .
$$

Rearranging term, the platform problem becomes

$$
\begin{aligned}
& \Pi^{C T}=-\kappa \alpha+\frac{1}{r+\gamma} \max \left\{\Pi_{1}^{C T}, \Pi_{2}^{C T}\right\}, \text { where } \\
& \Pi_{1}^{C T} \equiv \max _{\rho_{t}} G_{1}^{C T}\left(\rho_{t}\right) \text { s.t. } i \leq(1-\alpha) \rho_{t} ; \\
& \Pi_{2}^{C T} \equiv \max _{\rho_{t}} G_{2}^{C T}\left(\rho_{t}\right) \text { s.t. } i>(1-\alpha) \rho_{t},
\end{aligned}
$$



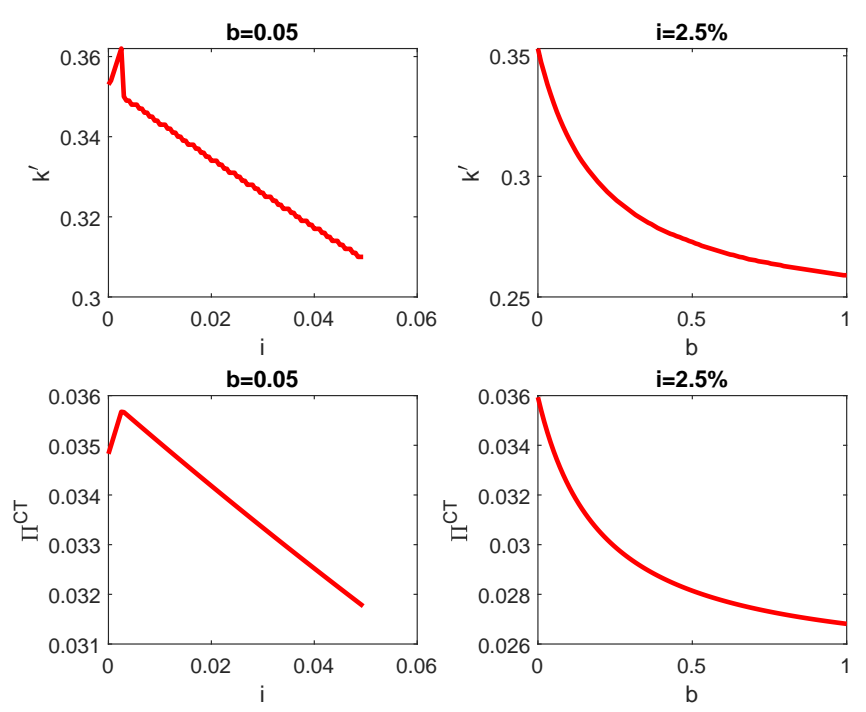

Figure 2: Supply of Circulatable Tokens

where $G_{1}^{C T}(\rho) \equiv\left[\rho-\frac{i b}{1-\gamma(1-b)}\right]\left(1+\frac{\rho-i}{\sigma \alpha}\right)^{-1 / \eta}$ and $G_{2}^{C T}(\rho) \equiv\left[\rho-\frac{i b}{1-\gamma(1-b)}\right]\left(1+\frac{\rho}{\sigma}\right)^{-1 / \eta}$.

We use a numerical example to illustrate the incentives of a platform to make tokens circulatable. Figure 2 plots the supply of circulatable tokens $k^{\prime}$ and profits $\Pi^{C T}$ as a function of $i$ and $b$. Whenever $b>0$, the platform needs to keep cash reserves which involve a marginal cost $i$. As a result, both token issuance and profits go down as the reserve ratio $b$ increases. The interest rate has non-linear effects. When $i$ is low, increasing $i$ makes cash a worse payment alternative outside the platform, allowing the platform to issue more tokens and make a higher profit. When $i$ is too high, further increasing $i$ will lower token issuance as a platform's profits decline with $i$. Figure 3 shows the platform's business model choice between issuing non-circulatable tokens, circulatable tokens and using cash. Under this parameterization, we find that a cash platform is profit-maximizing only for sufficiently low $i$ and sufficiently high $b$. The platform issues non-circulatable tokens when $i$ or $b$ are sufficiently high. In other cases, issuing circulatable tokens maximizes profits. In particular, in the absence of the minimum reserve requirement, a platform either accepts cash (for low $i$ ) or issues circulatable tokens (for high $i$ ).

\subsection{Calibrated examples}

We use the model to study two calibrated examples.

\section{Example 1. Cash platform: Amazon}

We consider an annual model and set $\beta=0.97$. We pick $\mu$ to generate $i=1.5 \%$ which is the 3-month T-bill rate in 2020 Q1. We set $\sigma=1$ to minimize matching frictions. With Amazon exemplifying the modeled cash platform in our model, we calibrate the trading parameter $\alpha$ and preference parameter $\eta$ to match the market share and trading fees of this e-commerce platform. First, for each item sold on their platform, Amazon charges the seller a referral fee which is a 


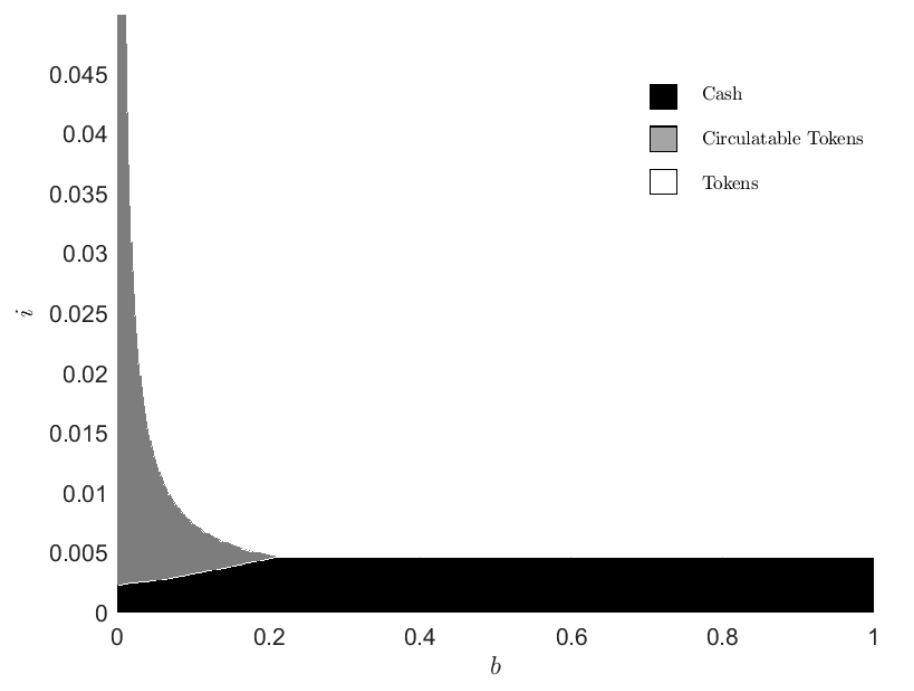

Figure 3: Platform Choice

flat percentage typically about $15 \% .^{13}$ Second, according to the Department of Commerce, the e-commerce share of retail in 2020 Q1 is $12 \%$. Since Amazon accounts for about $50 \%$ of the US e-commerce market, its market share is about $6 \% .{ }^{14}$ We set $\alpha=0.12$ and $\eta=0.13$ to minimize the sum of squared percentage differences (see Table 2).

Under our parameterization, $i^{*}=11 \%$. Since $i<i^{*}$, we are in case (i) considered in Proposition 3. This implies that it is not profitable for the platform to issue tokens for any $\kappa$ and $\gamma$. Suppose the setup cost of the token system is $\kappa=0.03$ (about $1 \%$ of $y^{T}$ ) and the probability of collapse is $1 \%$. Our model then suggests that it is socially optimal not to issue tokens for any $\gamma$. However, when $i$ goes above $12 \%$ or when $\alpha$ goes above $90 \%$, issuing tokens maximizes both profits and social welfare. As shown in Section 5, this is because high inflation constrains a cash platform's ability to charge fees, especially when its market share is large. When there is no reserve requirement, the platform will find it profitable to issue circulatable tokens for when $\kappa<1.8$. Hence the model suggests that the regulatory costs in the U.S. (captured by $\kappa$ or $b$ ) must be sufficiently high to prevent the platform from issuing circulatable tokens.

\section{Example 2. Circulatable tokens: Alibaba}

We now use the model to study the case of Alibaba in China. As shown in Table 3, we maintain the same preference parameter $\eta=0.13$ and set a small $\gamma=1 \%$ to capture the reliability of the platform. We pick $\mu$ to generate $i=3.5 \%$, which is the average 3 -month T-bill rate in China in 2017. We set $\sigma=1$ to minimize the matching frictions and set $\alpha=0.11$ to match the fact that Alibaba accounts for about $11 \%$ of China's total retail market. With a higher interest rate in China, the platform has a higher incentive to issue tokens, but issuing a non-circulatable token is still unprofitable. Issuing a circulatable token is profitable when $\kappa$ and $b$ are sufficiently low. For

\footnotetext{
${ }^{13}$ Source: https://www.junglescout.com/blog/amazon-fba-fees

${ }^{14}$ Source: https://tinyurl.com/y4xsyz6b
} 
Table 2: Parameter Values for Example 1

\begin{tabular}{lcll}
\hline \hline Parameters & Notation & Value & Notes \\
\hline Discount factor & $\beta$ & 0.97 & Standard in literature \\
Nominal interest rate & $i$ & $1.5 \%$ & 3-month T-bill rate in 2020 \\
Prob. of trading & $\sigma$ & 1 & Minimal search fractions \\
Prob. of platform trades & $\alpha$ & 0.12 & Amazon share of retail share \\
Preference parameter & $\eta$ & 0.13 & Amazon fee 15\% \\
\hline \hline
\end{tabular}

example, when $b=0$, the threshold fixed cost is $\kappa=5.2$; when $b=0.5$, the threshold fixed cost is $\kappa=2.3$; when $b=0.99$, the threshold fixed cost becomes $\kappa=0.02$. Hence the model suggests that the regulatory costs in China must be sufficiently low to induce Alibaba to issue circulable tokens in 2004. Indeed, some researchers have argued that the success of Alipay in China was due to the very tolerant financial regulation: Alipay was introduced in 2004, but it did not obtain the official payment license until 2011 (Huang, 2020). Our numerical exercise suggests that, if there had been a $100 \%$ reserve requirement from the start, the platform could have chosen not to issue tokens.

Another reason for Alipay's success is the fact that China's electronic payment infrastructure was under-developed before the introduction of Alipay. Compared to the U.S. and other developed economies, credit cards, debit cards, and bank cards were much less used in China. As a result, the introduction of Alipay helped facilitate transactions that were previously infeasible. ${ }^{15}$ Here we slightly modify the model to illustrate this idea. Suppose, due to payment frictions, cash payments can only be used for fraction $\delta<1$ of transactions on the platform. In contrast, tokens can be used to facilitate all transactions on the platform. Hence the introduction of tokens can expand the extensive margin of platform transactions. Suppose $\delta=0.86$, then the platform has an incentive to issue non-circulatable tokens whenever $\kappa<0.18 .{ }^{16}$ Hence, introducing tokens is more profitable when it helps overcome payment frictions relative to existing payment instruments.

Overall, this numerical exercise identifies three potential explanations for the differences between Amazon and Alibaba. First, the opportunity cost of holding cash is higher in China (captured by $i$ ). Second, the regulatory costs are lower in China (captured by $b, \kappa$ ). Third, the payment infrastructure was under-developed in China before the introduction of Alipay (captured by $\delta$ ).

\section{Conclusion}

We develop a general equilibrium model to study a platform's business model choice between running a cash platform and a token platform, as well as the welfare and policy implications of this choice. We show that the equilibrium business model choice is not necessarily socially

\footnotetext{
${ }^{15}$ For example, Alibaba provided its first payment service when a Chinese student had difficulty paying a seller in Japan to buy a second-hand camera in 2003 (Huang, 2020).

${ }^{16}$ This parameter choice is motivated by the fact that about $80 \%$ of Chinese adults have a bank account in 2017 . The corresponding number in the U.S. is $93 \%$. We normalize the value of $\sigma$ for the U.S. to 1 .
} 
Table 3: Parameter Values for Example 2

\begin{tabular}{lccl}
\hline \hline Parameters & Notation & Value & Notes \\
\hline Discount factor & $\beta$ & 0.97 & Standard in literature \\
Nominal interest rate & $i$ & $3.5 \%$ & 3-month Treasury bond rate in 2017 \\
Prob. of trading & $\sigma$ & 1 & Minimal search fractions \\
Prob. of platform trades & $\alpha$ & 0.11 & Alibaba accounts for 11\% of total retail in China \\
Preference parameter & $\eta$ & 0.13 & Same as that in Example 1 \\
\hline \hline
\end{tabular}

optimal because the private platform does not internalize its impacts on the economy, especially the spillover effects on non-platform transactions. We also extend the model to endogenize payment interoperability. The model suggests that the need to impose a reserve requirement depends on the nature of the risk associated with the platform, as well as the platform's market share and the central bank monetary policy.

While our paper has provided novel economic and policy insights, we have abstracted from some interesting aspects. When the platform can exert private efforts to reduce the cyber risk, there is a moral hazard in the choice of platform security. Reserve holdings can potentially increase the platform's "skin in the game," implying that imposing a minimum reserve requirement could sometimes be welfare enhancing. One could also endogenize the platform's monopoly power by endogenizing the network effect via the entry of merchants or studying the competition between multiple private platforms. It would also be interesting to explore other policy tools, such as the monetary policy of the central bank. Also, offering payment services can have other benefits to the platform. For example, "Big Tech" platforms can profit from the acquisition and monetization of user data (Chiu and Koeppl, 2020). We leave these extensions for future research. 


\section{References}

Andolfatto, David, "Assessing the impact of central bank digital currency on private banks," FRB St. Louis Working Paper 2018-25, 2018.

Auer, Raphael, Giulio Cornelli, and Jon Frost, "Rise of the central bank digital currencies: Drivers, approaches and technologies," BIS Working Paper, 2020, (880).

Barrdear, John and Michael Kumhof, "The macroeconomics of central bank issued digital currencies," Bank of England Working Paper, 2016.

Biais, Bruno, Christophe Bisiere, Matthieu Bouvard, and Catherine Casamatta, "The blockchain folk theorem," The Review of Financial Studies, 2019, 32 (5), 1662-1715.

, and Albert J Menkveld, "Equilibrium bitcoin pricing," Available at SSRN 3261063, 2020.

Brunnermeier, Markus K, Harold James, and Jean-Pierre Landau, "The digitalization of money," National Bureau of Economic Research Working Paper, 2019.

Catalini, Christian and Joshua S Gans, "Initial coin offerings and the value of crypto tokens," National Bureau of Economic Research Working Paper, 2018.

Chiu, Jonathan and Thorsten Koeppl, "Payments and the D(ata) N(etwork) A(ctivities) of BigTech platforms," 2020.

and Thorsten V Koeppl, "The economics of cryptocurrencies: Bitcoin and beyond," Bank of Canada Staff Working Paper 2019-40, 2019.

and Tsz-Nga Wong, "E-money: Efficiency, stability and optimal policy," Bank of Canada Working Paper 2014-16, 2014.

and __ , "On the essentiality of e-money," Bank of Canada Staff Working Paper 2015-43, 2015 .

, Mohammad Davoodalhosseini, Janet Hua Jiang, and Yu Zhu, "Bank market power and central bank digital currency: Theory and quantitative assessment," Bank of Canada Staff Working Paper 2019-20, 2019.

Choi, Michael and Guillaume Rocheteau, "Money mining and price dynamics," American Economic Journal Macroeconomics, 2020, forthcoming.

Cong, Lin William, Ye Li, and Neng Wang, "Tokenomics: Dynamic adoption and valuation," The Review of Financial Studies, 2020, forthcoming.

Davoodalhosseini, Seyed Mohammadreza, "Central bank digital currency and monetary policy," Bank of Canada Staff Working Paper 2018-36, 2018.

Easley, David, Maureen O'Hara, and Soumya Basu, "From mining to markets: The evolution of bitcoin transaction fees," Journal of Financial Economics, 2019, 134 (1), 91-109.

Eyal, Ittay and Emin Gün Sirer, "Majority is not enough: Bitcoin mining is vulnerable," in "International Conference on Financial Cryptography and Data Security" Springer 2014, pp. $436-454$.

Fernández-Villaverde, Jesús, Daniel Sanches, Linda Schilling, and Harald Uhlig, "Central bank digital currency: Central banking for all?," Review of Economic Dynamics, 2020. 
Gans, Joshua S and Hanna Halaburda, "Some economics of private digital currency," Economic analysis of the digital economy, 2015 Apr 20, pp. 257-276.

Garratt, Rodney and Maarten RC Van Oordt, "Entrepreneurial incentives and the role of initial coin offerings," Bank of Canada Staff Working Paper 2019-18, 2019.

Howell, Sabrina T, Marina Niessner, and David Yermack, "Initial coin offerings: Financing growth with cryptocurrency token sales," The Review of Financial Studies, 2020, 33 (9), 3925-3974.

Huang, Yiping, "FinTech Development in the People's Republic of China and Its Macroeconomic Implications," 2020.

Huberman, Gur, Jacob Leshno, and Ciamac C Moallemi, "Monopo ly without a monopolt: An economic analysis of the bitcoin payment system," Bank of Finland Research Discussion Paper, 2017, (27).

Kahn, Charles M and William Roberds, "Credit and identity theft," Journal of Monetary Economics, 2008, 55 (2), 251-264.

and __ "Why pay? An introduction to payments economics," Journal of Financial Intermediation, 2009, 18 (1), 1-23.

_ Francisco Rivadeneyra, and Tsz-Nga Wong, "Eggs in One Basket: Security and Convenience of Digital Currencies," FRB St. Louis Working Paper 2020-032, 2020. , and __ "Should the central bank issue e-money?," Journal of Financial Market Infrastructures, 2020, 8 (4), 1-22.

Keister, Todd and Daniel R Sanches, "Should central banks issue digital currency?," FRB Philadelphia Working Paper 19-26, 2019.

Lagos, Ricardo and Randall Wright, "A unified framework for monetary theory and policy analysis," Journal of political Economy, 2005, 113 (3), 463-484.

Li, Yiting, "Currency and checking deposits as means of payment," Review of Economic Dynamics, 2011, 14 (2), 403-417.

Monnet, Cyril and William Roberds, "Optimal pricing of payment services," Journal of Monetary Economics, 2008, 55 (8), 1428-1440.

Pagnotta, Emiliano, "Decentralizing Money: Bitcoin Prices and Blockchain Security," The Review of Financial Studies, 2020, forthcoming.

Saleh, Fahad, "Blockchain without waste: Proof-of-stake," The Review of Financial Studies, 2020, forthcoming.

Schilling, Linda and Harald Uhlig, "Some simple bitcoin economics," Journal of Monetary Economics, 2019, 106, 16-26.

Shy, Oz and Zhu Wang, "Why do payment card networks charge proportional fees?," American Economic Review, 2011, 101 (4), 1575-1590.

Sockin, Michael and Wei Xiong, "A model of cryptocurrencies," National Bureau of Economic Research Working Paper, 2020.

Williamson, Stephen, "Central bank digital currency: Welfare and policy implications," Unpub- 
lished, University of Western Ontario, 2019.

Wong, Tsz-Nga, "A tractable monetary model under general preferences," The Review of Economic Studies, 2016, 83 (1), 402-420.

You, Yang and Kenneth S Rogoff, "Redeemable Platform Currencies," National Bureau of Economic Research Working Paper, 2019.

Zhu, Yu and Scott Hendry, "A Framework for Analyzing Monetary Policy in an Economy with E-money," Bank of Canada Staff Working Paper 2019-01, 2019. 


\section{Appendix}

\section{A Proof of Lemma 1}

Proof. Note first that, given $\tau_{t} \leq 1$ and $\eta<1$, constraint (2) is always binding, implying that we must have $q_{t}=m_{t}$. If (2) were not binding then we have $m_{t}^{-\eta}<1$, which implies $\left(1-\tau_{t}\right)^{1-\eta} m_{t}^{-\eta}<1$ and hence the RHS of (6) is zero, contradicting the LHS which is strictly positive.

We then consider the two possible cases for equation (6): (i) $\left\{\left(1-\tau_{t}\right)^{1-\eta} m_{t}^{-\eta}-1\right\}_{+}=0$, and (ii) $\left\{\left(1-\tau_{t}\right)^{1-\eta} m_{t}^{-\eta}-1\right\}_{+}>0$. In case (i), equation (6) becomes $i=\sigma(1-\alpha)\left(m_{t}^{-\eta}-1\right)$, so we have $m_{t}=[1+i /[\sigma(1-\alpha)]]^{-1 / \eta}=\left[1-\tau_{C}(i)\right]^{1 / \eta-1}$. Since constraint (3) is not binding in case (i), using equation (5) the first order condition of (4) with respect to $y_{t}$ implies that $y_{t}=\left(1-\tau_{t}\right)^{1 / \eta} \leq\left(1-\tau_{t}\right) m_{t}$.

In case (ii), equation (6) becomes $i=\sigma(1-\alpha)\left(m_{t}^{-\eta}-1\right)+\sigma \alpha\left[\left(1-\tau_{t}\right)^{1-\eta} m_{t}^{-\eta}-1\right]$, so we have $m_{t}=m\left(\tau_{t}\right)$. Since constraint (3) is binding in case (ii), we have $y_{t}=\left(1-\tau_{t}\right) q_{t}$.

\section{B Proof of Proposition 1}

We first establish the following lemma, which derives some properties of the objective functions in these two regions.

Lemma 5 We have

(i) $G_{1}(\tau) \leq G_{2}(\tau)$ if and only if $\tau \geq \tau_{C}(i)$;

(ii) $G_{1}(\tau)$ is single peaked at $\tau=\eta ; G_{2}(\tau)$ is single peaked at $\tau=\bar{\tau}$, where $\bar{\tau} \in(\eta, 1)$.

Proof. Part (i) is obvious after arranging terms of $G_{1}(\tau)$ and $G_{2}(\tau)$. To show part (ii), differentiating $\log G_{1}(\tau)$ we have

$$
\frac{d}{d \tau} \log G_{1}(\tau)=\frac{1}{\tau}-\left(\frac{1}{\eta}-1\right) \frac{1}{1-\tau},
$$

which is strictly decreasing from infinity to negative infinity when $\tau$ goes from 0 to 1 , attaining zero at $\tau=\eta$. So $G_{1}(\tau)$ is single peaked at $\tau=\eta$. Differentiating $\log G_{2}(\tau ; i)$ we have

$$
\frac{d}{d \tau} \log G_{2}(\tau)=\frac{1}{\tau}-\frac{1-\eta}{\eta} \frac{\alpha(1-\tau)^{-\eta}}{1-\alpha+\alpha(1-\tau)^{1-\eta}},
$$

which is strictly decreasing from infinity to negative infinity when $\tau$ goes from 0 to 1 , attaining zero at $\tau=\bar{\tau}$. So $G_{2}(\tau)$ is single peaked at $\tau=\bar{\tau}$. Finally, note that $\bar{\tau}$ solves the FOC of $G_{2}(\tau)$, which is $1=(1-\bar{\tau})^{-\eta}(\bar{\tau} / \eta-1) /(1 / \alpha-1)$. Evaluating the FOC at $\tau=\eta$ and $\tau=1$ we have 0 and $\infty$. So $\bar{\tau}$ must lie within $(\eta, 1)$.

Given the results of Lemma 5, now we are ready to prove Proposition 1.

Proof. Consider the following three cases (the possible ranking of the $\tau$ threshold and the two peaks): (a) $\tau_{C}(i)<\eta<\bar{\tau}$; (b) $\tau_{C}(i) \in[\eta, \bar{\tau}]$; (c) $\tau_{C}(i)>\bar{\tau}>\eta$.

Consider case (a). Since $G_{2}(\tau ; i)$ is single peaked at $\tau=\bar{\tau}$ but $\bar{\tau}>\tau_{C}(i)$, we must have $\max _{\tau_{t} \leq \tau_{C}(i)} G_{2}\left(\tau_{t} ; i\right)=G_{2}\left[\tau_{C}(i)_{t} ; i\right]$. Notice that

$$
\Pi_{2}^{C}=\max _{\tau_{t} \leq \tau_{C}(i)} G_{2}\left(\tau_{t} ; i\right)=G_{2}\left[\tau_{C}(i) ; i\right]=G_{1}\left[\tau_{C}(i)\right]<\max _{\tau_{t} \geq \tau_{C}(i)} G_{1}\left(\tau_{t}\right)=\Pi_{1}^{C} .
$$


Thus we have $\Pi^{C}=\frac{\alpha \sigma}{r+\gamma} G_{1}(\eta)$ and $\tau=\eta$. This corresponds to case i of Lemma 1 , and hence $q_{t}=\left[1-\tau_{C}(i)\right]^{1 / \eta-1}$ and $y_{t}=(1-\eta)^{1 / \eta}$. Rearranging terms, we have $\tau_{C}(i)<\eta$ is equivalent to $i<i^{*}$, corresponding to part (a) of Proposition 1.

Consider case (b). Since $G_{1}(\tau)$ is single peaked at $\tau=\eta$ but $\eta \leq \tau_{C}(i)$, we must have $\max _{\tau_{t} \geq \tau_{C}(i)} G_{1}\left(\tau_{t}\right)=G_{1}\left[\tau_{C}(i)\right]$. Notice that

$$
\Pi_{2}^{C}=\max _{\tau_{t} \leq \tau_{C}(i)} G_{2}\left(\tau_{t} ; i\right)=G_{2}\left[\tau_{C}(i) ; i\right]=G_{1}\left[\tau_{C}(i)\right]=\max _{\tau_{t} \geq \tau_{C}(i)} G_{1}\left(\tau_{t}\right)=\Pi_{1}^{C} .
$$

Thus we have $\tau=\tau_{C}(i)$ and $\Pi^{C}=\frac{\alpha \sigma}{r+\gamma} G_{1}\left[\tau_{C}(i)\right]$. This corresponds to case i of Lemma 1, and hence $q_{t}=\left[1-\tau_{C}(i)\right]^{1 / \eta-1}$ and $y_{t}=\left[1-\tau_{C}(i)\right]^{1 / \eta}$. Rearranging terms, we have $\tau_{C}(i) \in[\eta, \bar{\tau}]$ is equivalent to $i \in\left[i^{*}, \hat{\imath}\right]$, corresponding to part (b) of Proposition 1.

Consider case $(\mathrm{c})$. The premise of case $(\mathrm{c})$ implies that

$$
\Pi_{1}^{C}=\max _{\tau_{t} \geq \tau_{C}(i)} G_{1}\left(\tau_{t}\right)=G_{1}\left[\tau_{C}(i)\right]=G_{2}\left[\tau_{C}(i) ; i\right]<\max _{\tau_{t} \leq \tau_{C}(i)} G_{2}\left(\tau_{t} ; i\right)=\Pi_{2}^{C} .
$$

Thus we have $\tau=\bar{\tau}$ and $\Pi^{C}=\frac{\alpha \sigma}{r+\gamma} G_{2}(\bar{\tau} ; i)$. This corresponds to case i of Lemma 1 , and hence $q_{t}=m\left[\tau_{C}(i)\right]$ and $y_{t}=(1-\bar{\tau}) q_{t}$. Rearranging terms, we have $\tau_{C}(i)>\bar{\tau}$ is equivalent to $i>\hat{\imath}$, corresponding to part (c) of Proposition 1.

\section{Proof of Proposition 2}

Proof. We omit the proof of the comparative statics of $i$ and $\gamma$ as they are tedious but straightforward. Given the definition of $\tau_{C}(i)$, we have

$$
\frac{\partial \tau_{C}(i)}{\partial \alpha}>0
$$

Notice that

$$
\begin{aligned}
& \left.\frac{\partial^{2} G_{2}(\tau ; i)}{\partial \alpha \partial \tau}\right|_{\tau=\bar{\tau}}=\left.G_{2}(\bar{\tau} ; i) \frac{\partial^{2}}{\partial \alpha \partial \tau} \log G_{2}(\tau ; i)\right|_{\tau=\bar{\tau}}=-\frac{1}{\alpha} \frac{(1-\alpha)(1-\eta)}{\bar{\tau}-\eta} G_{2}(\bar{\tau} ; i), \\
& \left.\frac{\partial^{2} G_{2}(\tau ; i)}{\partial^{2} \tau}\right|_{\tau=\bar{\tau}}=\left.G_{2}(\bar{\tau} ; i) \frac{\partial^{2}}{\partial \tau^{2}} \log G_{2}(\tau ; i)\right|_{\tau=\bar{\tau}}=-\frac{(1-\alpha)(1-\bar{\tau})+\eta[\bar{\tau}-\eta]}{\bar{\tau}^{2}(1-\eta)(1-\alpha)(1-\bar{\tau})} G_{2}(\bar{\tau} ; i) .
\end{aligned}
$$

Since $\bar{\tau}>\eta$ given by Lemma 5 , we have

$$
\frac{\partial \bar{\tau}}{\partial \alpha}=-\left.\frac{\partial^{2} G_{2}(\tau ; i)}{\partial \alpha \partial \tau}\right|_{\tau=\bar{\tau}} /\left.\frac{\partial^{2} G_{2}(\tau ; i)}{\partial^{2} \tau}\right|_{\tau=\bar{\tau}}<0 .
$$

In the case of $i<i^{*}, \tau_{t}$ and $y_{t}$ are independent to $\alpha$ so their comparative statics is zero. Given $q_{t}=\left[1-\tau_{C}(i)\right]^{1 / \eta-1}$ in this case, we have $\frac{\partial q_{t}}{\partial \alpha}<0$. Since $G_{1}(\eta)$ is independent to $\alpha$, given $\Pi^{C}=\frac{\alpha \sigma}{r+\gamma} G_{1}(\eta)$ in this case we have $\frac{\partial \Pi^{C}}{\partial \alpha}>0$.

In the case of $i \in\left[i^{*}, \hat{\imath}\right]$, note that the signs of the comparative statics of $q_{t}$ and $y_{t}$ are the same, which follows the comparative statics of $q_{t}$ in the case of $i<i^{*}$. Since we have $\eta \leq \tau_{C}(i)$ in the case of $i \in\left[i^{*}, \hat{\imath}\right]$ (see the proof of Proposition 1), we have $G_{1}^{\prime}\left[\tau_{C}(i)\right]<0$ as $\tau=\tau_{C}(i)$ is on the RHS of the single peak $\tau=\eta$ of $G_{1}(\tau)$. Applying (C.1) on $\Pi^{C}=\frac{\alpha \sigma}{r+\gamma} G_{1}\left[\tau_{C}(i)\right]$, we have $\frac{\partial \Pi^{C}}{\partial \alpha}$ ambiguous. 
In the case of $i>\hat{\imath}$, since $\bar{\tau}$ solves $G_{2}^{\prime}(\bar{\tau} ; i)=0$, we have

$$
\alpha=\left[1+(1-\bar{\tau})^{1-\eta}\left(\frac{\bar{\tau}}{\eta} \frac{1-\eta}{1-\bar{\tau}}-1\right)\right]^{-1}
$$

Substituting the above to $q_{t}$, we have

$$
\begin{aligned}
q_{t} & =\left(1+\frac{i}{\sigma}\right)^{-1 / \eta}\left[\frac{\bar{\tau}-\eta+\eta(1-\bar{\tau})^{\eta}}{\bar{\tau}(1-\eta)}\right]^{-1 / \eta}, \\
y_{t} & =\left(1+\frac{i}{\sigma}\right)^{-1 / \eta}(1-\bar{\tau})\left[\frac{\bar{\tau}-\eta+\eta(1-\bar{\tau})^{\eta}}{\bar{\tau}(1-\eta)}\right]^{-1 / \eta}
\end{aligned}
$$

where are non-monotone in $\bar{\tau}$, thus we have $\frac{\partial q_{t}}{\partial \alpha}, \frac{\partial y_{t}}{\partial \alpha}$, and $\frac{\partial \Pi^{C}}{\partial \alpha}$ ambiguous.

\section{Proof of Proposition 3}

Proof. After substituting $\rho_{t}$, the platform problem is a repetition of the following static problem:

$$
\Pi^{T}=-\kappa \alpha+\frac{\sigma \alpha}{r+\gamma} \max _{b_{t}, k_{t}, \tau_{t} \geq 0}\left\{\left[\left(1-\tau_{t}\right) k_{t}\right]^{1-\eta}+\left(1-\tau_{t}\right) k_{t}-\frac{i b_{t}}{1-\gamma\left(1-b_{t}\right)} k_{t}\right\} .
$$

The solution is simply $b_{t}=0$ and $\left(1-\tau_{t}\right) k_{t}=(1-\eta)^{1 / \eta}$. Substituting the above solution, we have proved Proposition 3.

\section{E Proof of Proposition 4}

Proof. We omit the proof as it is straightforward from Proposition 3.

\section{F Proof of Proposition 5}

Proof. Combining (21) and (23) cash is used on the platform if

$$
y_{t}^{-\eta}-1 \geq \frac{\tau_{t}^{M}}{1-\tau_{t}^{M}}
$$


Substituting $\rho_{t}$ of (21) into (24), the platform problem is

$$
\begin{aligned}
\Pi^{D} & =-\kappa \alpha+\max \left\{\sum_{t=1}^{\infty}(1-\gamma)^{t-1} \beta^{t}\left\{\begin{array}{c}
\sigma \alpha\left(y_{t}^{-\eta}-1\right) y_{t}^{K}-\frac{i b_{t}}{1-\gamma\left(1-b_{t}\right)} k_{t} \\
+\sigma \alpha \frac{\tau_{t}^{M}}{1-\tau_{t}^{M}}\left(y_{t}-y_{t}^{K}\right)
\end{array}\right\}\right. \text {, s.t.(22) and (F.1) } \\
& \leq-\kappa \alpha+\max \left\{\sum_{t=1}^{\infty}(1-\gamma)^{t-1} \beta^{t}\left\{\begin{array}{c}
\sigma \alpha\left(y_{t}^{-\eta}-1\right) y_{t}-\frac{i b_{t}}{1-\gamma\left(1-b_{t}\right)} k_{t} \\
-\sigma \alpha\left[\left(y_{t}^{-\eta}-1\right)-\frac{\tau_{t}^{M}}{1-\tau_{t}^{M}}\right]\left(y_{t}-y_{t}^{K}\right)
\end{array}\right\}\right\} \text {, s.t.(22) and (F.1) } \\
& \leq-\kappa \alpha+\max \left\{\sum_{t=1}^{\infty}(1-\gamma)^{t-1} \beta^{t}\left\{\sigma \alpha\left(y_{t}^{-\eta}-1\right) y_{t}-\frac{i b_{t}}{1-\gamma\left(1-b_{t}\right)} k_{t}\right\}\right\} \text {, s.t. (F.1) } \\
& \leq \Pi^{T},
\end{aligned}
$$

where the second inequality follows (F.1). If (F.1) is not satisfied, then the dual platform is simply a token platform, thus we have $\Pi^{D}=\Pi^{T}$. If (21) holds with strict inequality, then the dual platform is simply a cash platform, thus we have $\Pi^{D}=\Pi^{C}$.

\section{G Proof of Lemma 2}

Proof. Denote $\Delta \Pi(i) \equiv\left(\Pi^{C}-\Pi^{T}\right) / \alpha$. Using Proposition 1 and Proposition 3 , we have

$$
\Delta \Pi(i)=\left\{\begin{array}{l}
\kappa \text { if } i<i^{*} \\
\kappa+\frac{\sigma}{r+\gamma}\{\underbrace{\left[1-\left[1+\frac{i}{\sigma(1-\alpha)}\right]^{-1 /(1-\eta)}\right]\left[1+\frac{i}{\sigma(1-\alpha)}\right]^{-1 / \eta}-\underbrace{\eta(1-\eta)^{1 / \eta}}_{G_{1}(\eta)}\} \text { if } i \in\left[i^{*}, \hat{\imath}\right]}_{G_{1}\left[\tau_{C}(i)\right]} \\
\kappa+\frac{\sigma}{r+\gamma}[\underbrace{\left(1+\frac{i}{\sigma}\right)^{-1 / \eta} \bar{\tau}\left[1-\alpha+\alpha(1-\bar{\tau})^{1-\eta}\right]^{1 / \eta}}_{G_{2}(\bar{\tau} ; i)}-\underbrace{\eta(1-\eta)^{1 / \eta}}_{G_{1}(\eta)}] \text { if } i>\hat{\imath} .
\end{array}\right.
$$

Notice that $\Delta \Pi(0)>0$ and $\Delta \Pi(\infty)<0$. Using Proposition 2 , we have $\partial \Delta \Pi(i) / \partial i \leq 0$ (strict inequality when $\left.i>i^{*}\right)$, so there exist a unique $\iota^{\Pi}$ such that $\Delta \Pi\left(\iota^{\Pi}\right)=0$ and $\Pi^{C}<\Pi^{T}$ if and only if $i>\iota^{\Pi}$. Notice that

$$
\frac{\partial \Delta \Pi(i)}{\partial \alpha}=\left\{\begin{array}{l}
0 \text { if } i<i^{*} \\
\frac{\partial \Pi^{C}}{\partial i} \frac{\alpha}{1-\alpha}<0 \text { if } i \in\left[i^{*}, \hat{\imath}\right] \\
-\frac{\sigma \bar{\tau}\left(1+\frac{i}{\sigma}\right)^{-1 / \eta}}{\eta(r+\gamma)}\left[1-\alpha+\alpha(1-\bar{\tau})^{1-\eta}\right]^{1 / \eta-1}\left[1-(1-\bar{\tau})^{1-\eta}\right]<0 \text { if } i>\hat{\imath} .
\end{array}\right.
$$

Using $\frac{\partial \iota^{\Pi}}{\partial \alpha}=-\frac{\partial \Delta \Pi\left(\iota^{\Pi}\right)}{\partial \alpha} / \frac{\partial \Delta \Pi\left(\iota^{\Pi}\right)}{\partial i} \leq 0$ (strict inequality when $i>i^{*}$ ), we have proved that $\iota$ is decreasing in $\alpha$. Finally, notice that $G_{1}(\eta)>G_{1}\left[\tau_{C}(i)\right]>G_{2}(\bar{\tau} ; i)$, we have $\partial \Delta \Pi(i) / \partial \gamma \geq 0$ (strict inequality when $i>i^{*}$ ) and hence $\frac{\partial \iota^{\Pi}}{\partial \gamma}=-\frac{\partial \Delta \Pi\left(\iota^{\Pi}\right)}{\partial \gamma} / \frac{\partial \Delta \Pi\left(\iota^{\Pi}\right)}{\partial i} \geq 0$. 


\section{H Proof of Proposition 6}

Proof. We consider the three cases in proposition 1. In case (a), a token platform is not adopted. In case (b), $y^{C}<y^{T}$, according to propositions 1 to 3 . Also, $q^{C}=q^{T}=\left[1-\tau_{C}(i)\right]^{1 / \eta-1}$. In case (c), $y^{C}<y^{T}$, according to lemma 1 to 3. Also, $q^{C}=m(\bar{\tau})$ and $q^{T}=\left[1-\tau_{C}(i)\right]^{1 / \eta-1}$ in this case. At $i=\hat{\imath}$, we have $m(\bar{\tau})=\left[1-\tau_{C}(\hat{\imath})\right]^{1 / \eta-1}$. For $i>\hat{\imath}$, we have $m(\bar{\tau})$ fixed and $\left[1-\tau_{C}(i)\right]^{1 / \eta-1}$ declining in $i$. Therefore, we have $q^{C} \geq q^{T}$ in this case. The third statement is obvious given the defination of $\mathcal{S}$ and the result that reserve is $b=0$.

\section{Proof of Lemma 3}

Proof. Define $\Delta \mathcal{W}(i) \equiv\left(\mathcal{W}^{C}-\mathcal{W}^{T}\right) / \alpha$. Recall that the allocations under the cash and token platforms are given by

\begin{tabular}{l|cccc} 
& $\tau$ & $q_{t}^{C}$ & $q_{t}^{T}$ & $y_{t}^{C}$ \\
\hline (a). $i<i^{*}$ & $\eta$ & {$\left[1-\tau_{C}(i)\right]^{1 / \eta-1}$} & {$\left[1-\tau_{C}(i)\right]^{1 / \eta-1}$} & $(1-\eta)^{1 / \eta}$ \\
(b). $i \in\left[i^{*}, \hat{\imath}\right]$ & $\tau_{C}(i)$ & {$\left[1-\tau_{C}(i)\right]^{1 / \eta-1}$} & {$\left[1-\tau_{C}(i)\right]^{1 / \eta-1}$} & {$\left[1-\tau_{C}(i)\right]^{1 / \eta}$} \\
(c). $i>\hat{\imath}$ & $\bar{\tau}$ & $m(\bar{\tau})$ & {$\left[1-\tau_{C}(i)\right]^{1 / \eta-1}$} & $(1-\bar{\tau}) q_{t}$ \\
& $y_{t}^{T}$ & $\Pi^{C}$ & \multicolumn{1}{c}{$\Pi^{T}$} \\
\hline (a). $i<i^{*}$ & $(1-\eta)^{1 / \eta}$ & $\frac{\alpha \sigma}{r+\gamma} G_{1}(\eta)$ & $-\frac{\kappa \alpha}{r+\gamma}+\frac{\sigma \alpha \eta}{r+\gamma}(1-\eta)^{1 / \eta-1}$ \\
(b). $i \in\left[i^{*}, \hat{\imath}\right]$ & $(1-\eta)^{1 / \eta}$ & $\frac{\alpha \sigma}{r+\gamma} G_{1}\left[\tau_{C}(i)\right]$ & $-\frac{\kappa \alpha}{r+\gamma}+\frac{\sigma \alpha \eta}{r+\gamma}(1-\eta)^{1 / \eta-1}$ \\
(c). $i>\hat{\imath}$ & $(1-\eta)^{1 / \eta}$ & $\frac{\alpha \sigma}{r+\gamma} G_{2}(\bar{\tau} ; i)$ & $-\frac{\kappa \alpha}{r+\gamma}+\frac{\sigma \alpha \eta}{r+\gamma}(1-\eta)^{1 / \eta-1}$.
\end{tabular}

Using Proposition 1 and Proposition 3, we have

$$
\Delta \mathcal{W}(i)=\left\{\begin{array}{l}
\kappa \text { if } i<i^{*} \\
\kappa+\frac{\sigma}{r+\gamma}\left\{u\left(y^{C}\right)-y^{C}-u\left(y^{T}\right)+y^{T}\right\} \text { if } i \in\left[i^{*}, \hat{\imath}\right] ; \\
\kappa+\frac{\sigma}{r+\gamma}\left\{u\left(y^{C}\right)-y^{C}-u\left(y^{T}\right)+y^{T}\right\} \\
+\frac{\sigma}{r+\gamma}(1 / \alpha-1)\left\{u\left(q^{C}\right)-q_{t}^{C}-u\left(q^{T}\right)+q^{T}\right\}
\end{array} \quad \text { if } i>\hat{\imath} .\right.
$$

In the case of $i \in\left[i^{*}, \hat{\imath}\right]$, it is straightforward to see that $\frac{\partial \Delta \mathcal{W}(i)}{\partial i}<0$. In the case of $i>\hat{\imath}$, differentiating the above and using the fact that $y_{t}^{C}=(1-\bar{\tau}) q_{t}^{C}$ from Proposition 1, we have

$$
\frac{\partial \Delta \mathcal{W}(i)}{\partial i}\left\{\begin{array}{l}
\leq 0 \text { if } i \leq \hat{\imath} \\
=\frac{i}{\alpha(r+\gamma)}\left[\left(1+\frac{\sigma \alpha \bar{\tau}}{i}\right) \frac{\partial q^{C}}{\partial i}-\frac{\partial q^{T}}{\partial i}\right] \text { if } i>\hat{\imath}
\end{array}\right.
$$

For the derivative of $i$, we have

$$
\left(1+\frac{\sigma \alpha \bar{\tau}}{i}\right) \frac{\partial q^{C}}{\partial i}-\frac{\partial q^{T}}{\partial i}=\frac{1}{\eta \sigma(1-\alpha)}\left(1+\frac{i}{\sigma(1-\alpha)}\right)^{-1 / \eta-1} H(i)
$$

where

$$
H(i)=1+\frac{\sigma \alpha \bar{\tau}}{i}-(1-\alpha)^{-1 / \eta}\left(1-\frac{\alpha}{1+\frac{i}{\sigma}}\right)^{1 / \eta+1}\left[1-\alpha+\alpha(1-\bar{\tau})^{1-\eta}\right]^{1 / \eta}
$$


The sign of $\frac{\partial \Delta \mathcal{W}_{t}(i)}{\partial i}$ in the case of $i>\hat{\imath}$ depends on $H(i)$, which is decreasing in $i$. Notice that

$$
H(\hat{\imath}) \equiv \alpha+\frac{\sigma \alpha \bar{\tau}}{\hat{\imath}}>0, H(\infty)=1-\left[1+\frac{\alpha(1-\bar{\tau})^{1-\eta}}{1-\alpha}\right]^{1 / \eta}<0
$$

Also, $\frac{\partial \Delta \mathcal{W}(i)}{\partial i}=\beta^{-1} \kappa \alpha>0$ and $\frac{\partial \Delta \mathcal{W}(i)}{\partial i} \propto-\Pi^{T}<0$, so there exists a unique $\iota^{\mathcal{W}}$ such that $\left.\frac{\Delta \mathcal{W}(i)}{\partial i}\right|_{i=\iota} \mathcal{W}=0$ and $\mathcal{W}^{T}<\mathcal{W}^{C}$ if and only if $i<\iota^{\mathcal{W}}$. For the derivative of $\alpha$, using Proposition 1 and Proposition 3 we have

$$
\frac{\partial \Delta \mathcal{W}(i)}{\partial \alpha} \leq 0 \text { if } i \leq \hat{\imath} .
$$

Thus we have $\frac{\partial \iota^{\mathcal{W}}}{\partial \alpha} \equiv-\frac{\partial \Delta \mathcal{W}\left({ }_{\iota} \mathcal{W}\right)}{\partial \alpha} / \frac{\partial \Delta \mathcal{W}\left(\iota^{\mathcal{W}}\right)}{\partial i}<0$ if $i \leq \hat{\imath}$. For the derivative of $\gamma$, notice that

$$
\frac{\partial \Delta \mathcal{W}\left(\iota^{\mathcal{W}}\right)}{\partial \gamma}=-\left[\left.\begin{array}{c}
\frac{\sigma}{(r+\gamma)^{2}}\left\{u\left(y^{C}\right)-y^{C}-u\left(y^{T}\right)+y^{T}\right\} \\
+\frac{\sigma(1 / \alpha-1)}{(r+\gamma)^{2}}\left\{u\left(y^{C}\right)-q^{C}-u\left(q^{T}\right)+q^{T}\right\}
\end{array}\right|_{i={ }_{\iota} \mathcal{W}}\right]=\frac{\kappa}{r+\gamma}>0
$$

Thus we have $\frac{\partial \iota \mathcal{W}}{\partial \gamma} \equiv-\frac{\partial \Delta \mathcal{W}\left(\iota^{\mathcal{W}}\right)}{\partial \gamma} / \frac{\partial \Delta \mathcal{W}\left({ }_{\iota} \mathcal{W}\right)}{\partial i}>0$

\section{J Proof of Proposition 7}

Proof. The repeated static problem is reduced to

$$
\Pi^{T}=-\kappa \alpha+\frac{\sigma \alpha}{r+\gamma} \max _{b_{t} \geq \bar{b}, y_{t}, \tau_{t} \geq 0}\left\{y_{t}^{1-\eta}-\left[1+\frac{\gamma \tau_{t}+\frac{i}{\sigma \alpha}}{1-\tau_{t}} \frac{b_{t}}{1-\gamma\left(1-b_{t}\right)}\right] y_{t}\right\},
$$

where the solution is simply $b_{t}=\bar{b}$ and $\tau_{t}=0$. The first-order condition with respect to $y_{t}$ implies

$$
y_{t}=\left[\frac{1-\eta}{1+\frac{i}{\sigma \alpha} \frac{\bar{b}}{1-\gamma(1-\bar{b})}}\right]^{1 / \eta}
$$

Substituting the above solution, we have proved Proposition 7.

\section{K Proof of Lemma 4}

Proof. Consider two cases of (27): in the first case $\left(m_{t}>0\right)$, we have $i=\sigma(1-\alpha)\left\{\left(m_{t}+k_{t}\right)^{-\eta}-1\right\}_{+}$, and in the second case $\left(m_{t}=0\right)$ we have $i>\sigma(1-\alpha)\left\{\left(m_{t}+k_{t}\right)^{-\eta}-1\right\}_{+}$. In the first case, we have $m_{t}+k_{t}=\{1+i /[\sigma(1-\alpha)]\}^{-1 / \eta}$ and $k_{t}^{-\eta} \geq\left(m_{t}+k_{t}\right)^{-\eta}=1+i /[\sigma(1-\alpha)]>1$. Thus, we 
have $\rho_{t}=\sigma\left[\alpha\left(k_{t}^{-\eta}-1\right)+\frac{i}{\sigma}\right]$, and hence $k_{t}=\left(1+\frac{\rho_{t}-i}{\sigma \alpha}\right)^{-1 / \eta}$. The premise $m_{t}>0$ is satisfied if

$$
m_{t}+k_{t}=\left[1+\frac{i}{\sigma(1-\alpha)}\right]^{-1 / \eta}>k_{t}=\left(1+\frac{\rho_{t}-i}{\sigma \alpha}\right)^{-1 / \eta} \Leftrightarrow i \leq(1-\alpha) \rho_{t} .
$$

In the second case, rearranging terms, we have $k_{t}^{-\eta}<1+i /[\sigma(1-\alpha)]$. Thus, we have $k_{t}=$ $\left(1+\frac{\rho_{t}}{\sigma}\right)^{-1 / \eta}$. 


\section{K.1 Online Appendix: Deposit insurance}

To deal with the collapse risk, a commonly used regulation is deposit insurance. One can treat the platform as a bank and tokens as deposits. Suppose the central bank requires a token platform to pay a premium at the rate $\varphi$ if the cyber shock does not hit. When the shock realizes, the insurance will compensate token holders for the loss of the token value. Since the token holders are fully insured against the cyber risk, the nominal price of retail goods in terms of tokens is equal to that in terms of cash, i.e., $P_{t}^{T}=P_{t}=1 / \phi_{t}$. In this case, it is obvious that the platform never holds reserves $\left(b_{t}=0\right)$, because the loss is fully covered by deposit insurance. The shadow interest rate of tokens becomes

$$
\rho_{t}=\sigma \alpha\left\{\left(1-\tau_{t}\right)^{1-\eta} k_{t}^{-\eta}-1\right\}_{+} .
$$

The initial profit flow of the platform in stage 2 is

$$
x_{0}=p_{0} K_{1}-\kappa \alpha .
$$

The expected profit flow in the latter period is

$$
\bar{x}_{t}=(1-\gamma) p_{t}\left[K_{t+1}-K_{t}-\varphi K_{t}\right]+\sigma \alpha \frac{\tau_{t} \phi_{t} P_{t}^{T} y_{t}}{1-\tau_{t}}
$$

The platform's profit is given by $\Pi^{D}=\max \left\{x_{0}+\sum_{t=1}^{\infty}(1-\gamma)^{t-1} \beta^{t} \bar{x}_{t}\right\}$. By collecting terms of the same $t$ across periods, the platform's profit can be expressed as

$$
\begin{aligned}
\Pi^{D}=-\kappa \alpha+\max _{k_{t}, \tau_{t} \geq 0}\left\{\begin{array}{l}
p_{0} K_{1} \\
+\sum_{t=1}^{\infty}(1-\gamma)^{t-1} \beta^{t}(1-\gamma) \frac{p_{t}}{p_{t+1}} p_{t+1} K_{t+1} \\
+\sum_{t=1}^{\infty}(1-\gamma)^{t-1} \beta^{t}\left[-(1-\gamma)(1+\varphi) p_{t} K_{t}+\sigma \alpha \frac{\tau_{t} \phi_{t} P_{t}^{T} y_{t}}{1-\tau_{t}}\right]
\end{array}\right\} \\
=-\kappa \alpha+\max _{k_{t}, \tau_{t} \geq 0}\left\{\sum_{t=1}^{\infty}(1-\gamma)^{t-1} \beta^{t}\left[1+\rho_{t}-(1-\gamma)(1+\varphi)+\sigma \alpha \tau_{t}\right] k_{t}\right\},
\end{aligned}
$$

where the platform takes as given the premium rate $\varphi$. In equilibrium, the premium rate of the deposit insurance is actuarially fair, such that

$$
(1-\gamma) \varphi=\gamma
$$

We assume that the insurance premium (and payout after the cyber shock) are rebated to consumers as lump sum transfers.

By substituting $y_{t}=\left(1-\tau_{t}\right) k_{t}$, the repeated static problem is reduced to

$$
\max _{y_{t}, \tau_{t} \geq 0}\left\{y_{t}^{1-\eta}-y_{t}\right\}
$$

which is independent to $\tau_{t}$. Thus, $\tau_{t}$ is indeterminate, as long as $\rho_{t} \geq 0$, which implies $\tau_{t} \in[0, \eta]$. The first order condition with respect to $y_{t}$ implies

$$
y_{t}=(1-\eta)^{1 / \eta} .
$$

Substituting the above solution, we can derive the following proposition. 
Proposition 8 The platform is indifferent to any $\tau_{t} \in[0, \eta]$. The equilibrium allocation of a token platform is the same, i.e., the deposit insurance does not affect the equilibrium allocation and profits of the token platform, i.e.,

$$
\begin{aligned}
\rho_{t} & =\sigma \alpha \frac{\eta-\tau_{t}}{1-\eta} \\
q_{t} & =\left[1-\tau_{C}(i)\right]^{1 / \eta-1} \\
y_{t} & =(1-\eta)^{1 / \eta} \\
\Pi^{D} & =-\kappa \alpha+\frac{\sigma \alpha \eta}{r+\gamma}(1-\eta)^{1 / \eta-1} .
\end{aligned}
$$

Corollary 3 The deposit insurance does not affect welfare but slows down the growth rate of the nominal token price $g$.

Here is why deposit insurance is neutral in this economy. Recall that the platform can always self-insure against the cyber risk by holding reserves $b$. Deposit insurance is cheaper than holding reserves because buying deposit insurance does not incur the opportunity cost of nominal interest $i$. However, this cheaper insurance option is not useful for the platform because the platform chooses to hold zero reserves in the absence of deposit insurance. Instead, the platform compensates the consumers' expected loss from the cyber risk by engineering a higher token price growth $g_{t}$. Thus, the rise of insurance premium is offset one-to-one by the corresponding decrease in $g_{t}$, while the profits and allocation stay unchanged. 